\title{
A Constructive Algebraic Strategy for Interpolatory Subdivision Schemes Induced by Bivariate Box splines
}

\author{
Costanza Conti, Luca Gemignani $†$ Lucia Romani ${ }^{\ddagger}$
}

September 21, 2012

\begin{abstract}
This paper describes an algebraic construction of bivariate interpolatory subdivision masks induced by three-directional box spline subdivision schemes. Specifically, given a three-directional box spline, we address the problem of defining a corresponding interpolatory subdivision scheme by constructing an appropriate correction mask to convolve with the three-directional box spline mask. The proposed approach is based on the analysis of certain polynomial identities in two variables and leads to interesting new interpolatory bivariate subdivision schemes.
\end{abstract}

Key words: Interpolatory subdivision schemes, Subdivision symbols, Bezout equation, Bivariate polynomial, Matrix polynomial, Box splines.

2010 Mathematics Subject Classification: 65F05; 68W30; 65D05; 41A15

\section{Introduction}

Interpolatory subdivision schemes are useful practical tools to generate graphs of functions, curves and surfaces interpolating given sets of points. As any subdivision scheme, they consist in the repeated application of linear rules determining successive refinements of coarse initial meshes and converging to continuous (interpolatory) limits. In the "standard" bivariate situation, the refinement rules are simple average rules with the average coefficients defining the so called subdivision mask, a finite sequence of real numbers hereafter denoted by $\mathbf{a}=\left\{a_{\alpha}, \alpha \in \mathbb{Z}^{2}\right\}$. In the interpolatory case, the mask a satisfies $a_{2 \alpha}=\delta_{\alpha, 0}$, for all $\alpha \in \mathbb{Z}^{2}$. The subdivision mask $\mathbf{a}$ is associated with the bivariate Laurent polynomial, or subdivision symbol,

$$
a\left(z_{1}, z_{2}\right)=\sum_{\alpha \in \mathbb{Z}^{2}} a_{\alpha} z^{\alpha}, \quad z^{\alpha}=z_{1}^{\alpha_{1}} z_{2}^{\alpha_{2}}, \quad z_{1}, z_{2} \in \mathbb{C},
$$

whose algebraic properties translate into analytical properties of the corresponding subdivision scheme and of its limit (see [2], [3], [10], [21] and references therein). For example, a convergent subdivision scheme is interpolatory if and only if its symbol satisfies

$$
a\left(z_{1}, z_{2}\right)+a\left(-z_{1}, z_{2}\right)+a\left(z_{1},-z_{2}\right)+a\left(-z_{1},-z_{2}\right)=4,
$$

or a "shifted" version of it.

*Dipartimento di Energetica "Sergio Stecco", Università di Firenze, Viale Morgagni 40/44, 50134 Firenze, Italy. tel.: +39-0554796713 - fax: +39-0554224137 - email: costanza.conti@unifi.it

${ }^{\dagger}$ Dipartimento di Matematica, Università di Pisa, Largo Bruno Pontecorvo 5, 56127 Pisa, Italy. tel.: +39-0502213291 - fax: +39-0502213224 - email: gemignan@dm.unipi.it

${ }^{\ddagger}$ Dipartimento di Matematica e Applicazioni, Università di Milano-Bicocca, Via R. Cozzi 53, 20125 Milano, Italy. tel.: +39-0264485735 - fax: +39-0264485705 - email: lucia.romani@unimib.it 
This is actually the feature that we are going to exploit in this paper to provide an algebraic procedure for constructing an interpolatory bivariate symbol from a given non-interpolatory one. In particular, in this paper we confine our attention to the case of a three-directional box spline symbol i.e.,

$$
a\left(z_{1}, z_{2}\right)=4 \frac{\left(1+z_{1}\right)^{k_{1}}}{2^{k_{1}}} \frac{\left(1+z_{2}\right)^{k_{2}}}{2^{k_{2}}} \frac{\left(1+z_{1} z_{2}\right)^{k_{3}}}{2^{k_{3}}}, \quad k_{i}>0, i=1,2,3,
$$

since, in view of the results in [5], three-directional symbols are building blocks of any reasonable bivariate subdivision scheme. This subject was started in [19] and further developed in [20] where interpolatory subdivision masks induced by bivariate box splines were considered for the first time. There, the regularity and the interpolatory property of the underlying refinable function as well as the convergence of the corresponding subdivision scheme are established only for three-directional box splines of type $B_{k, k, k}$ belonging to the smoothness class $C^{2 k-2}$. Later, the same problem was investigated by Jia in [15], where existence and uniqueness of interpolatory masks induced by multidimensional box splines of type $B_{k, k, k}$ is discussed, though no constructive strategies are given. In this paper, a family of interpolatory masks induced by a more general box spline $B_{k_{1}, k_{2}, k_{3}}$ is considered.

At the core of our approach is the idea of looking for a bivariate polynomial correction $m\left(z_{1}, z_{2}\right)$ such that the modified symbol $\hat{a}\left(z_{1}, z_{2}\right)=a\left(z_{1}, z_{2}\right) m\left(z_{1}, z_{2}\right)$ fulfills the necessary condition $\hat{a}(1,1)=4$ and the interpolatory condition

$$
\hat{a}\left(z_{1}, z_{2}\right)+\hat{a}\left(-z_{1}, z_{2}\right)+\hat{a}\left(z_{1},-z_{2}\right)+\hat{a}\left(-z_{1},-z_{2}\right)=4 z_{1}^{n_{1}} z_{2}^{n_{2}},
$$

for suitable even integers $n_{1}, n_{2}$. More specifically, our strategy may determine a family of bivariate polynomial corrections

$$
m_{\omega}\left(z_{1}, z_{2}\right)=m\left(z_{1}, z_{2}\right)+\omega k\left(z_{1}, z_{2}\right), \quad \omega \in \mathbb{R},
$$

with $m\left(z_{1}, z_{2}\right)$ as above and $k\left(z_{1}, z_{2}\right)$ a "kernel" polynomial such that $k(1,1)=0$ and $\tilde{a}\left(z_{1}, z_{2}\right)=$ $a\left(z_{1}, z_{2}\right) k\left(z_{1}, z_{2}\right)$ satisfies

$$
\tilde{a}\left(z_{1}, z_{2}\right)+\tilde{a}\left(-z_{1}, z_{2}\right)+\tilde{a}\left(z_{1},-z_{2}\right)+\tilde{a}\left(-z_{1},-z_{2}\right)=0 .
$$

In one respect this paper is therefore a continuation of [6], [7] where the unidimensional case is treated. In fact, in [6], [7] it is shown that, under some reasonable assumptions on the degree of the correction polynomials and under some primality assumptions on the symbol of the given univariate non-interpolatory scheme, all the admissible corrections can be determined by solving a set of polynomial equations that are equivalent in a matrix setting to inverting a certain Hurwitz matrix defined by the coefficients of the given symbol. But, on the other hand, it is clear that the bivariate extension of the results in [6], [7] is not straightforward at all, since the solution of the analogue bivariate polynomial equation through the use of Hilbert Nullstellensatz generally provides high degree solutions of poor applicative value.

To circumvent this difficulty, in this paper we follow a different approach motivated by the work of Rabarison and de Villiers [12] on the characterization of interpolatory bivariate mask symbols. The proposed strategy proceeds by splitting the correction symbol $m\left(z_{1}, z_{2}\right)$ into two components which can be individually characterized as solutions of Bezout-like equations for univariate polynomials with coefficients over an integral domain. The results in [6], [7] can thus be applied for both the analysis of the equations and possibly the computation of the components. Also, the introduction of the real parameter $\omega$ and of the family of kernel polynomials $k\left(z_{1}, z_{2}\right)$ greatly extends the flexibility of the resulting algebraic construction by allowing the computation of solutions with specific requirements such as symmetry, low degree, polynomial reproduction, etc., that are important in applications. In other words specific values of the free parameter $\omega$ can be set in order the interpolatory subdivision scheme satisfies extra conditions than the one induced by the interpolatory mask. Moreover, our strategy can be easily extended to the case where a symbol factor is not of box spline type as, for example, to

$$
a\left(z_{1}, z_{2}\right)=4 \frac{\left(1+z_{1}\right)^{k_{1}}}{2^{k_{1}}} \frac{\left(1+z_{2}\right)^{k_{2}}}{2^{k_{2}}} b\left(z_{1}, z_{2}\right), \quad k_{i}>0, i=1,2,
$$


with $b(1,1)=1, b\left(-1 ; z_{2}\right) \neq 0$ and $b\left(z_{1},-1\right) \neq 0$ for at least one $z_{1}, z_{2} \in \mathbb{C}$, a case which is presently under investigation.

To conclude, we mention that, as in previous works, the complete convergence and regularity analysis of the subdivision scheme associated to the constructed interpolatory mask is here conducted a posteriori due to the non linearity of the condition the correction symbol has to satisfy. Nevertheless, the condition $m(1,1)=4$ guarantees, at least, the existence of an associated compactly supported distribution which is refinable with respect to the interpolatory mask. At last, we also point out that there are necessary and sufficient conditions for convergence and smoothness analysis of a subdivision scheme in terms of the given mask (see, [10], [14], [16], [17], [19], for example). Once we have introduced the algebraic machinery for the solution of (1.1) these techniques can be applied for the analisys of the resulting subdivision schemes as well as for the selection of specific solutions among the ones of the algebraic equation (1.1). Some examples of this approach are provided at the end of this paper where we essentially use the results in [10] for the convergence and smoothness analysis of some novel schemes.

The paper is organized as follows. Section 2 starts with a brief summary of the strategy proposed in [7] to determine admissible corrections for a univariate non-interpolatory symbol to become interpolatory. Then, it continues with the description of the construction of an interpolatory bivariate symbol induced by a three-directional box spline symbol. Applications to specific box spline symbols are illustrated in Section 3 where we show that the proposed algebraic construction leads to interesting new interpolatory bivariate subdivision schemes analyzed in full detail. Section 4 presents a further alternative constructive approach for the determination of the polynomial correction. Conclusions and future work are drawn in Section 5.

\section{The algebraic construction}

To make the paper self contained we start by briefly summarizing the univariate strategy proposed in [7] to determine admissible corrections for a non-interpolatory symbol to become interpolatory. The univariate results that we recall in the sequel will be repeatedly used in the bivariate construction.

\subsection{The univariate framework}

In [7] the set of minimal-degree univariate polynomials that are eligible to convert a non-interpolatory subdivision symbol $c(z) \in \mathbb{R}[z]$ into an interpolatory one is fully characterized. In particular, it is shown that these polynomials are obtained by solving a set of structured Bezout equations that are equivalent, in a matrix setting, to inverting a Hurwitz matrix defined by the coefficients of the given symbol.

Proposition 2.1. [7] Let $c(z)=c_{0}+c_{1} z+\ldots c_{k} z^{k} \in \mathbb{R}[z]$ be a polynomial of degree $k$ relatively prime with $c(-z)$. Let $\mathcal{H}^{-} \in \mathbb{R}^{k \times k}$ and $\mathcal{H}^{+} \in \mathbb{R}^{k \times k}$ be the Hurwitz matrices defined by

$$
\mathcal{H}^{-}:=\left[\begin{array}{cccccc}
c_{1} & c_{0} & 0 & \ldots & \ldots & \ldots \\
c_{3} & c_{2} & c_{1} & c_{0} & 0 & \ldots \\
c_{5} & c_{4} & c_{3} & \ldots & & \ldots \\
\vdots & \vdots & \vdots & \vdots & \ldots \\
\vdots & \vdots & \vdots & \vdots & \ldots \\
c_{2(k-1)+1} & c_{2(k-1)} & c_{2(k-1)-1} & \ldots & & \ldots
\end{array}\right],
$$




$$
\mathcal{H}^{+}:=\left[\begin{array}{ccccc}
c_{0} & 0 & \ldots & \ldots & \ldots \\
c_{2} & c_{1} & c_{0} & 0 & \ldots \\
c_{4} & c_{3} & c_{2} & \ldots & \ldots \\
\vdots & \vdots & \vdots & \vdots & \ldots \\
\vdots & \vdots & \vdots & \vdots & \ldots \\
c_{2(k-1)} & c_{2(k-1)-1} & c_{2(k-1)-2} & \ldots & \ldots
\end{array}\right],
$$

where $c_{j}=0$ if $j>k$. Then $\mathcal{H}^{-}$and $\mathcal{H}^{+}$are invertible and, moreover, the polynomials $p_{i}^{-}(z)$ and $p_{i}^{+}(z)$, with coefficients given by the entries of the $i$-th column $(1 \leq i \leq k)$ of $\left(\mathcal{H}^{-}\right)^{-1}$ and $\left(\mathcal{H}^{+}\right)^{-1}$ are respectively the unique polynomials of degree less than $k$ such that

$$
c(z) p_{i}^{-}(z)-c(-z) p_{i}^{-}(-z)=2 z^{2 i-1}, \quad 1 \leq i \leq k,
$$

and

$$
c(z) p_{i}^{+}(z)+c(-z) p_{i}^{+}(-z)=2 z^{2 i-2}, \quad 1 \leq i \leq k .
$$

Observe that the solution of the Bezout equations (2.1) and (2.2) is a linear problem which is customarily reformulated into a matrix setting as the solution of a Sylvester resultant linear system of order $2 k$. For the very special case considered here the $k \times k$ Hurwitz matrices $\mathcal{H}^{-}$and $\mathcal{H}^{+}$encompass the properties of the Sylvester-based formulation by providing a simplified approach. This can also be extended to deal with polynomials having coefficients over an integral domain rather than a field.

\subsection{The bivariate framework}

Let us start investigating the problem of constructing a bivariate interpolatory symbol given the bivariate polynomial associated with the (approximating) three-directional subdivision symbol

$$
a\left(z_{1}, z_{2}\right)=2^{2-k_{1}-k_{2}-k_{3}}\left(1+z_{1}\right)^{k_{1}}\left(1+z_{2}\right)^{k_{2}}\left(1+z_{1} z_{2}\right)^{k_{3}}, \quad z_{1}, z_{2} \in \mathbb{C},
$$

for some integers $k_{1}, k_{2}, k_{3} \in \mathbb{N}$. Our problem consists of finding (if it exists) a correction $m\left(z_{1}, z_{2}\right)$ that is a bivariate polynomial such that the modified symbol

$$
\hat{a}\left(z_{1}, z_{2}\right)=a\left(z_{1}, z_{2}\right) m\left(z_{1}, z_{2}\right)
$$

fulfills the interpolatory condition

$$
\hat{a}\left(z_{1}, z_{2}\right)+\hat{a}\left(-z_{1}, z_{2}\right)+\hat{a}\left(z_{1},-z_{2}\right)+\hat{a}\left(-z_{1},-z_{2}\right)=4 z_{1}^{n_{1}} z_{2}^{n_{2}},
$$

for suitable even integers $n_{1}$ and $n_{2}$. As we are interested in finding a correction of degree (in some specified sense) as low as possible, we impose the additional condition that $0 \leq n_{1} \leq 2\left(k_{1}-1\right)$ and $0 \leq n_{2} \leq 2\left(k_{2}-1\right)$. Such interval ranges may be translated by considering shifted solutions. The modified symbol $\hat{a}\left(z_{1}, z_{2}\right)$ can also be expressed as

$$
\hat{a}\left(z_{1}, z_{2}\right)=2^{2-k_{1}-k_{2}}\left(1+z_{1}\right)^{k_{1}}\left(1+z_{2}\right)^{k_{2}} \hat{b}\left(z_{1}, z_{2}\right),
$$

where

$$
\hat{b}\left(z_{1}, z_{2}\right)=\frac{\left(1+z_{1} z_{2}\right)^{k_{3}}}{2^{k_{3}}} m\left(z_{1}, z_{2}\right) .
$$

Let us consider the polynomial $h\left(z_{1}, z_{2}\right)$ defined by

$$
h\left(z_{1}, z_{2}\right)=\hat{a}\left(z_{1}, z_{2}\right)+\hat{a}\left(z_{1},-z_{2}\right), \quad z_{1}, z_{2} \in \mathbb{C} .
$$

From (2.4) it follows that this polynomial should satisfy

$$
h\left(z_{1}, z_{2}\right)+h\left(-z_{1}, z_{2}\right)=4 z_{1}^{n_{1}} z_{2}^{n_{2}}, \quad z_{1}, z_{2} \in \mathbb{C} .
$$


In addition, from (2.5) we obtain that

$$
h\left(z_{1}, z_{2}\right)=2^{2-k_{1}-k_{2}}\left(1+z_{1}\right)^{k_{1}}\left\{\left(1+z_{2}\right)^{k_{2}} \hat{b}\left(z_{1}, z_{2}\right)+\left(1-z_{2}\right)^{k_{2}} \hat{b}\left(z_{1},-z_{2}\right)\right\},
$$

which gives

$$
h\left(z_{1}, z_{2}\right)=2^{2-k_{1}-k_{2}}\left(1+z_{1}\right)^{k_{1}} g\left(z_{1}, z_{2}\right), \quad z_{1}, z_{2} \in \mathbb{C},
$$

where

$$
g\left(z_{1}, z_{2}\right)=\left(1+z_{2}\right)^{k_{2}} \hat{b}\left(z_{1}, z_{2}\right)+\left(1-z_{2}\right)^{k_{2}} \hat{b}\left(z_{1},-z_{2}\right) .
$$

By combining (2.7) and (2.6) we find that

$$
2^{-k_{1}-k_{2}}\left(1+z_{1}\right)^{k_{1}} g\left(z_{1}, z_{2}\right)+2^{-k_{1}-k_{2}}\left(1-z_{1}\right)^{k_{1}} g\left(-z_{1}, z_{2}\right)=z_{1}^{n_{1}} z_{2}^{n_{2}} .
$$

Since $c\left(z_{1}\right)=\left(1+z_{1}\right)^{k_{1}}$ and $c\left(-z_{1}\right)=\left(1-z_{1}\right)^{k_{1}}$ are relatively prime, we know from Subsection 2.1 that there exists a unique univariate polynomial $p_{0}\left(z_{1}\right) \in \mathbb{R}\left[z_{1}\right]$ of degree less than $k_{1}$ such that

$$
2^{-k_{1}-k_{2}}\left(1+z_{1}\right)^{k_{1}} p_{0}\left(z_{1}\right)+2^{-k_{1}-k_{2}}\left(1-z_{1}\right)^{k_{1}} p_{0}\left(-z_{1}\right)=z_{1}^{n_{1}} .
$$

The general solution of $(2.9)$ can thus be obtained from the solution $p_{0}\left(z_{1}\right)$ of $(2.10)$ by linearity. In fact we observe that the bivariate polynomial $\hat{g}\left(z_{1}, z_{2}\right)=g\left(z_{1}, z_{2}\right)-z_{2}^{n_{2}} p_{0}\left(z_{1}\right)$ satisfies the homogeneous equation

$$
2^{-k_{1}-k_{2}}\left(1+z_{1}\right)^{k_{1}} \hat{g}\left(z_{1}, z_{2}\right)+2^{-k_{1}-k_{2}}\left(1-z_{1}\right)^{k_{1}} g\left(-z_{1}, z_{2}\right)=0 \text {, }
$$

which says that

$$
\hat{g}\left(z_{1}, z_{2}\right)=g\left(z_{1}, z_{2}\right)-z_{2}^{n_{2}} p_{0}\left(z_{1}\right)=\left(1-z_{1}\right)^{k_{1}} t\left(z_{1}, z_{2}\right),
$$

where $t\left(z_{1}, z_{2}\right)$ is any bivariate polynomial which is odd in $z_{1}$, that is,

$$
t\left(z_{1}, z_{2}\right)+t\left(-z_{1}, z_{2}\right)=0 .
$$

By expressing $t\left(z_{1}, z_{2}\right)$ as a univariate polynomial in $z_{2}$ whose coefficients are polynomials in $z_{1}$, it is shown that this property implies that these coefficients are also odd polynomials. The proof immediately follows by using interpolation techniques and the identity principle for univariate polynomials. By replacing (2.11) into (2.8) we find that

$$
z_{2}^{n_{2}} p_{0}\left(z_{1}\right)+\left(1-z_{1}\right)^{k_{1}} t\left(z_{1}, z_{2}\right)=\left(1+z_{2}\right)^{k_{2}} \hat{b}\left(z_{1}, z_{2}\right)+\left(1-z_{2}\right)^{k_{2}} \hat{b}\left(z_{1},-z_{2}\right) .
$$

It is easy to see that the polynomial on the right hand side of (2.12) is even in $z_{2}$, and, therefore, since $n_{2}$ is an even integer, the same property should be fulfilled by $t\left(z_{1}, z_{2}\right)$. Summing up, $t\left(z_{1}, z_{2}\right)$ is a bivariate polynomial which is odd in $z_{1}$ and even in $z_{2}$ and, hence, it can be expressed as

$$
t\left(z_{1}, z_{2}\right)=\sum_{j=1}^{\ell} p_{j}\left(z_{1}\right) z_{2}^{2(j-1)}
$$

where $p_{j}\left(z_{1}\right), 1 \leq j \leq \ell$, are suitable odd polynomials and $\ell \in \mathbb{N}, \ell \geq k_{2}$.

Thus (2.12) can be rewritten as

$$
z_{2}^{n_{2}} p_{0}\left(z_{1}\right)+\left(1-z_{1}\right)^{k_{1}} \sum_{j=1}^{\ell} p_{j}\left(z_{1}\right) z_{2}^{2(j-1)}=\left(1+z_{2}\right)^{k_{2}} \hat{b}\left(z_{1}, z_{2}\right)+\left(1-z_{2}\right)^{k_{2}} \hat{b}\left(z_{1},-z_{2}\right) .
$$

The relation (2.13) is a specific instance for $z_{2}^{n_{2}} p_{0}\left(z_{1}\right)=\sum_{j=1}^{\ell} f_{j}\left(z_{1}\right) z_{2}^{2(j-1)}$ of the more general equation of the form

$$
\begin{aligned}
\sum_{j=1}^{\ell}\left(f_{j}^{(0)}\left(z_{1}\right)+(1\right. & \left.\left.-z_{1}\right)^{k_{1}} p_{j}\left(z_{1}\right)\right) z_{2}^{2(j-1)}= \\
& =\left(1+z_{2}\right)^{k_{2}}\left(1+z_{1} z_{2}\right)^{k_{3}} m\left(z_{1}, z_{2}\right)+\left(1-z_{2}\right)^{k_{2}}\left(1-z_{1} z_{2}\right)^{k_{3}} m\left(z_{1},-z_{2}\right)
\end{aligned}
$$

where $f_{j}^{(0)}\left(z_{1}\right)=f_{j}\left(z_{1}\right)$ are given prescribed polynomials. 
Remark 2.2. We observe that when $k_{1}, k_{2} \geq 1$ from (2.10) and $(2.13)$ follows that $\hat{b}(1,1)=1$. Moreover, if $f_{j}\left(z_{1}\right)=0$ (which is the case to get a "kernel" solution) we have $\hat{b}(1,1)=0$.

When the integer $\ell$ and the polynomials $p_{j}\left(z_{1}\right), 1 \leq j \leq \ell$ are fixed, then under some additional mild assumptions we can determine a solution $m\left(z_{1}, z_{2}\right)$ which is a polynomial in the variable $z_{2}$ with coefficients that are rational functions in the variable $z_{1}$. The main question to be addressed here is if for some suitable choices of $\ell$ and the polynomials $p_{j}\left(z_{1}\right), 1 \leq j \leq \ell$, these rational functions reduce to Laurent polynomials so that $m\left(z_{1}, z_{2}\right)$ becomes a bivariate Laurent polynomial. We summarize the ultimate computational task as follows:

Given the polynomials $f_{j}^{(0)}\left(z_{1}\right)=f_{j}\left(z_{1}\right), 1 \leq j \leq k_{2}$, we have to determine the integer $\ell \geq k_{2}$ and the odd polynomials $p_{j}\left(z_{1}\right), 1 \leq j \leq \ell$, such that (2.14) holds for a suitable bivariate Laurent polynomial $m\left(z_{1}, z_{2}\right)$. This solution is the candidate correction.

For solving (2.14) we can rely upon the natural isomorphism

$$
\mathbb{R}\left[z_{1}, z_{2}\right] \ni f\left(z_{1}, z_{2}\right) \rightarrow \hat{f}\left(z_{2}\right) \in \mathbb{R}\left[z_{1}\right]\left[z_{2}\right],
$$

mapping bivariate polynomials to univariate polynomials with (univariate) polynomial coefficients. Specifically, it is worth noting that $\left(1+z_{1} z_{2}\right)^{k_{3}}$ can be viewed as a univariate polynomial in $z_{2}$ of degree $k_{3}$ with coefficients in $\mathbb{R}\left[z_{1}\right]$, that is,

$$
\left(1+z_{1} z_{2}\right)^{k_{3}}=\sum_{i=0}^{k_{3}} b_{i}\left(z_{1}\right) z_{2}^{i}, \quad \text { with } \quad b_{i}\left(z_{1}\right)=\left(\begin{array}{c}
k_{3} \\
i
\end{array}\right) z_{1}^{i} .
$$

There follows that the univariate procedure described in Subsection 2.1 can in principle be applied to

$$
c\left(z_{2}\right)=\left(1+z_{2}\right)^{k_{2}}\left(1+z_{1} z_{2}\right)^{k_{3}}=\sum_{i=0}^{k_{2}+k_{3}} g_{i}\left(z_{1}\right) z_{2}^{i}
$$

where

$$
g_{i}\left(z_{1}\right)= \begin{cases}\sum_{j=0}^{i}\left(\begin{array}{c}
k_{2} \\
j
\end{array}\right)\left(\begin{array}{c}
k_{3} \\
i-j
\end{array}\right) z_{1}^{i-j}, & i=0, \cdots, \min \left\{k_{2}, k_{3}\right\} \\
\sum_{j=0}^{\infty}\left(\begin{array}{c}
k_{2} \\
j
\end{array}\right)\left(\begin{array}{c}
k_{3} \\
i-j
\end{array}\right) z_{1}^{i-j}, & i=\min \left\{k_{2}, k_{3}\right\}, \cdots, \max \left\{k_{2}, k_{3}\right\} \\
\sum_{j=i-k_{3}}^{k_{2}}\left(\begin{array}{c}
k_{2} \\
j
\end{array}\right)\left(\begin{array}{c}
k_{3} \\
i-j
\end{array}\right) z_{1}^{i-j}, & i=\max \left\{k_{2}, k_{3}\right\}, \cdots, k_{2}+k_{3}\end{cases}
$$

and the binomial coefficients in the second expression are interpreted in terms of the Gamma function. We continue by exploiting the association between the pair of univariate polynomials $c\left(z_{2}\right)$ and $c\left(-z_{2}\right)$ with the Hurwitz matrices $\mathcal{H}^{+}$and $\mathcal{H}^{-}$of order $n=k_{2}+k_{3}$, having entries over the integral domain $\mathbb{F}=\mathbb{R}\left[z_{1}\right]$. Namely, we have

$$
\mathcal{H}^{+}:=\left[\begin{array}{ccccc}
g_{0}\left(z_{1}\right) & 0 & \ldots & \ldots & \ldots \\
g_{2}\left(z_{1}\right) & g_{1}\left(z_{1}\right) & g_{0}\left(z_{1}\right) & 0 & \ldots \\
g_{4}\left(z_{1}\right) & g_{3}\left(z_{1}\right) & g_{2}\left(z_{1}\right) & \ldots & \ldots \\
\vdots & \vdots & \vdots & \vdots & \ldots \\
\vdots & \vdots & \vdots & \vdots & \ldots \\
g_{2\left(k_{2}+k_{3}-1\right)}\left(z_{1}\right) & g_{2\left(k_{2}+k_{3}-1\right)-1}\left(z_{1}\right) & g_{2\left(k_{2}+k_{3}-1\right)-2}\left(z_{1}\right) & \ldots & \ldots
\end{array}\right],
$$


and

$$
\mathcal{H}^{-}:=\left[\begin{array}{cccccc}
g_{1}\left(z_{1}\right) & g_{0}\left(z_{1}\right) & 0 & \ldots & \ldots & \ldots \\
g_{3}\left(z_{1}\right) & g_{2}\left(z_{1}\right) & g_{1}\left(z_{1}\right) & g_{0}\left(z_{1}\right) & 0 & \ldots \\
g_{5}\left(z_{1}\right) & g_{4}\left(z_{1}\right) & g_{3}\left(z_{1}\right) & \ldots & \ldots \\
\vdots & \vdots & \vdots & \vdots & \ldots \\
\vdots & \vdots & \vdots & \vdots & \ldots \\
g_{2\left(k_{2}+k_{3}-1\right)+1}\left(z_{1}\right) & g_{2\left(k_{2}+k_{3}-1\right)}\left(z_{1}\right) & g_{2\left(k_{2}+k_{3}-1\right)-1}\left(z_{1}\right) & \ldots & \ldots
\end{array}\right] .
$$

And so, virtually, we can think to attack the computation of $m\left(z_{1}, z_{2}\right)$ satisfying (2.14) by using Proposition 2.1 applied to this novel Hurwitz matrix $\mathcal{H}^{+} \in \mathbb{F}^{n \times n}$. The resulting approach can be summarized as follows:

\section{The basic idea of the correction polynomial construction}

1. Set $\ell=k_{2}+k_{3}=n$;

2. Solve $\mathcal{H}^{+} \boldsymbol{x}^{(j)}=\left(f_{j}^{(0)}\left(z_{1}\right)+\left(1-z_{1}\right)^{k_{1}} p_{j}\left(z_{1}\right)\right) \boldsymbol{e}_{j}, 1 \leq j \leq \ell$ with $f_{j}^{(0)}\left(z_{1}\right)$ and $p_{j}\left(z_{1}\right)$ specified above;

3. Define the polynomials $x_{j}\left(z_{2}\right)=\sum_{i=0}^{n-1} x_{i}^{(j)} z_{2}^{i}, 1 \leq j \leq \ell$;

4. Set $m\left(z_{1}, z_{2}\right)=\sum_{j=1}^{\ell} x_{j}\left(z_{2}\right)$.

It is worth observing that, from one hand, theoretical and computational problems can be encountered at step 2 where we have to solve a linear system defined by a polynomial matrix whose solutions generally lie in the quotient field of rational functions. On the other hand, the known vectors are partially undetermined since they depend on the unknown polynomials $p_{j}\left(z_{1}\right), 1 \leq j \leq \ell$. Our primary goal is to exploit these two issues simultaneously in such a way to find an eligible strategy for computing odd polynomials $p_{j}\left(z_{1}\right)$ corresponding with polynomial solutions of the system. In order to investigate the properties of this system we start by proving a result about the determinant of $\mathcal{H}^{+} \in \mathbb{F}^{n \times n}$. The proof follows by using a suitable generalization of the techniques employed in [7] to computations with matrices having entries over an integral domain. In particular we relate $\mathcal{H}^{+} \in \mathbb{F}^{n \times n}$ and $\mathcal{H}^{-} \in \mathbb{F}^{n \times n}$ with the Sylvester-resultant matrix $\mathcal{S} \in \mathbb{F}^{2 n \times 2 n}$ generated from the coefficients of $c\left(z_{2}\right)$ and $c\left(-z_{2}\right)$, that is,

$$
\mathcal{S}=\left[\begin{array}{cccccc}
g_{0}\left(z_{1}\right) & & & g_{0}\left(z_{1}\right) & & \\
g_{1}\left(z_{1}\right) & \ddots & & -g_{1}\left(z_{1}\right) & \ddots & \\
\vdots & & g_{0}\left(z_{1}\right) & \vdots & & g_{0}\left(z_{1}\right) \\
g_{n}\left(z_{1}\right) & & \vdots & (-1)^{n} g_{n}\left(z_{1}\right) & & \vdots \\
& \ddots & \vdots & & \ddots & \vdots \\
& & g_{n}\left(z_{1}\right) & & & (-1)^{n} g_{n}\left(z_{1}\right)
\end{array}\right] .
$$

Theorem 2.3. Let $c\left(z_{2}\right)$ as in (2.15) with $k_{2}, k_{3} \in \mathbb{N}$ and let $r\left(z_{1}\right)=\operatorname{res}\left(c\left(z_{2}\right), c\left(-z_{2}\right)\right)=\operatorname{det} \mathcal{S}$ be the resultant, i.e., the determinant of the Sylvester matrix of order $2\left(k_{2}+k_{3}\right)$, of $c\left(z_{2}\right)$ and $c\left(-z_{2}\right)$. We have that

$$
r\left(z_{1}\right)= \pm 2^{n} z_{1}^{k_{3}}\left(\operatorname{det}\left(\mathcal{H}^{+}\right)\right)^{2} .
$$


Proof. Let $P_{n} \in \mathbb{R}^{2 n \times 2 n}, P_{n}=\left(\delta_{i, \sigma(j)}\right)$ be the permutation matrix associated with the "perfect shuffle" permutation given by

$$
\sigma:\{1, \ldots, 2 n\} \rightarrow\{1, \ldots, 2 n\}, \quad \sigma(j)= \begin{cases}(j+1) / 2+n, & \text { if } j \text { is odd } \\ j / 2, & \text { if } j \text { is even }\end{cases}
$$

Furthermore, let $G_{n} \in \mathbb{R}^{2 n \times 2 n}$ be the matrix defined by

$$
G_{n}:=\left[\begin{array}{c|c}
I_{n} & -D_{n} \\
\hline D_{n} & I_{n}
\end{array}\right]
$$

where $D_{n}:=\operatorname{diag}\left[-1,(-1)^{2}, \ldots,(-1)^{n-1},(-1)^{n}\right]$. It is straightforward to verify that

$$
G_{n}^{-1}=\frac{1}{2}\left[\begin{array}{c|c}
I_{n} & D_{n} \\
\hline-D_{n} & I_{n}
\end{array}\right]
$$

By premultiplying the Sylvester-resultant matrix $\mathcal{S}$ by $P_{n}$ we find that

$$
P_{n} \mathcal{S}=\left[\begin{array}{c|c}
\mathcal{H}^{-} & \mathcal{H}^{-} D_{n} \\
\hline \mathcal{H}^{+} & -\mathcal{H}^{+} D_{n}
\end{array}\right]
$$

Hence, there follows that

$$
P_{n} \mathcal{S} G_{n}^{-1}=\left[\begin{array}{c|c}
0_{n} & \mathcal{H}^{-} D_{n} \\
\hline \mathcal{H}^{+} & 0_{n}
\end{array}\right] .
$$

From the Binet theorem this gives

$$
\pm r\left(z_{1}\right)\left(\frac{1}{2}\right)^{n}= \pm z_{1}^{k_{3}}\left(\operatorname{det}\left(\mathcal{H}^{+}\right)\right)^{2}
$$

which completes the proof.

Based on Theorem 2.3 we obtain an explicit expression for the determinant of $\mathcal{H}^{+} \in \mathbb{F}^{n \times n}$. First, we make use of a classical result in elimination theory to find an explicit expression for the resultant $r\left(z_{1}\right)=\operatorname{res}\left(c\left(z_{2}\right), c\left(-z_{2}\right)\right)$.

Theorem 2.4. Let $c\left(z_{2}\right)$ as in (2.15) with $k_{2}, k_{3} \in \mathbb{N}$. For the resultant $r\left(z_{1}\right)=\operatorname{res}\left(c\left(z_{2}\right), c\left(-z_{2}\right)\right) \in$ $\mathbb{R}\left[z_{1}\right]$, it holds that

$$
r\left(z_{1}\right)=\alpha z_{1}^{k_{3}^{2}}\left(1+z_{1}\right)^{2 k_{2} k_{3}},
$$

for a suitable $\alpha \in \mathbb{R} \backslash\{0\}$.

Proof. Setting $c\left(z_{2}\right)=\left(1+z_{2}\right)^{k_{2}}\left(1+z_{1} z_{2}\right)^{k_{3}}=f\left(z_{1}, z_{2}\right)$, by using well-known properties of the resultant polynomial [1], it is easy to see that

$$
\left\{z_{1} \in \mathbb{C}: r\left(z_{1}\right)=0\right\}=\left\{z_{1} \in \mathbb{C}: \exists z_{2} \in \mathbb{C}, f\left(z_{1}, z_{2}\right)=0=f\left(z_{1},-z_{2}\right)\right\} \cup\left\{z_{1} \in \mathbb{C}: g_{k_{2}+k_{3}}\left(z_{1}\right)=z_{1}^{k_{3}}=0\right\}
$$

where the roots are counted with their "multiplicity" which is the sum of the corresponding intersection multiplicities including points at infinity. From this characterization we deduce that the distinct roots of $r\left(z_{1}\right)$ are $z_{1}=0$ and $z_{1}=-1$. In order to evaluate the corresponding multiplicities we perform a perturbative analysis. Let us start considering the root $z_{1}=-1$. Observe that the polynomial system

$$
\left\{\begin{array}{l}
f\left(-1, z_{2}\right)=0 \\
f\left(-1,-z_{2}\right)=0
\end{array}\right.
$$


has two roots $z_{2}=1$ and $z_{2}=-1$. For the multiplicity of $(-1,1)$ we perform the substitution $z_{1}=-1+s$ and $z_{2}=1+t$, obtaining the system

$$
\left\{\begin{array}{l}
f_{1}(s, t)=f(-1+s, 1+t)=(2+t)^{k_{2}}[s(1+t)-t]^{k_{3}}=0 \\
f_{2}(s, t)=f(-1+s,-1-t)=(-1)^{k_{2}} t^{k_{2}}[2+t-s(1+t)]=0 .
\end{array}\right.
$$

A small perturbation of the coefficients of the polynomials yields the system

$$
\left\{\begin{array}{l}
\widetilde{f}_{1}(s, t)=(2+t)^{k_{2}}\left\{[s(1+t)-t]^{k_{3}}-\epsilon\right\}=0 \\
\widetilde{f}_{2}(s, t)=(-1)^{k_{2}}\left(t^{k_{2}}-\epsilon\right)[2+t-s(1+t)]=0,
\end{array}\right.
$$

where the common root at $s=t=0$ is spread out into $k_{2} k_{3}$ distinct roots and, therefore, $k_{2} k_{3}$ is the multiplicity of $(-1,1)$. A similar analysis applies to $(-1,-1)$, which implies that $2 k_{2} k_{3}$ is the multiplicity of $z_{1}=-1$. For the root $z_{1}=0$, which annihilates the leading coefficient $g_{k_{2}+k_{3}}\left(z_{1}\right)$ of $c\left(z_{2}\right)$, see $(2.15)$, we can proceed as follows. Let us introduce the perturbed polynomial

$$
\widetilde{f}\left(z_{1}, z_{2}\right)=\left(1+z_{2}\right)^{k_{2}}\left[\left(1+z_{1} z_{2}\right)^{k_{3}}-\epsilon z_{2}^{k_{3}}\right] .
$$

We find that the solutions of

$$
\left\{\begin{array}{l}
f\left(z_{1}, z_{2}\right)=0 \\
f\left(z_{1},-z_{2}\right)=0
\end{array}\right.
$$

at "infinite points" for $z_{1}=0$ are replaced by $k_{3}$ solutions of

$$
\left\{\begin{array}{l}
\widetilde{f}\left(z_{1}, z_{2}\right)=0 \\
\widetilde{f}\left(z_{1},-z_{2}\right)=0
\end{array}\right.
$$

given by $z_{1}^{(j)}=\epsilon^{k_{3}^{-1}} e^{2 \pi \sqrt{-1}(j-1) / k_{3}}, 1 \leq j \leq k_{3}$, each of them corresponding with $k_{3}$ distinct intersection points. In this way we conclude that the multiplicity of $z_{1}=0$ is $k_{3}^{2}$.

By virtue of Theorem 2.3 and Theorem 2.4, we obtain the desired expression of $\mathcal{H}^{+}$.

Corollary 2.5. For the matrix $\mathcal{H}^{+} \in \mathbb{F}^{n \times n}, \mathbb{F}=\mathbb{R}\left[z_{1}\right]$, generated from the coefficients of $c\left(z_{2}\right)=$ $\left(1+z_{2}\right)^{k_{2}}\left(1+z_{2} z_{3}\right)^{k_{3}}$, with $c\left(z_{2}\right)$ as in (2.15), it holds

$$
\operatorname{det} \mathcal{H}^{+}=\alpha z_{1}^{k_{3}\left(k_{3}-1\right) / 2}\left(1+z_{1}\right)^{k_{2} k_{3}},
$$

for a suitable $\alpha \in \mathbb{R} \backslash\{0\}$.

It is worth noting that the scaled polynomial

$$
h\left(z_{1}\right)=\frac{\operatorname{det} \mathcal{H}^{+}}{z_{1}^{k_{3}\left(k_{3}-1\right) / 2}}
$$

satisfies the following properties:

P.1 $h\left(z_{1}\right)$ and $h\left(-z_{1}\right)$ are relatively prime;

P.2 $h(0) h(1) \neq 0$.

These properties play a fundamental role to ensure the reliability of the four steps construction described above for computing $m\left(z_{1}, z_{2}\right)$ satisfying (2.14). The possibility of choosing the odd polynomials $p_{j}\left(z_{1}\right)$ freely is exploited in the following result.

Theorem 2.6. Let $f\left(z_{1}\right) \in \mathbb{R}\left[z_{1}\right]$ be a given polynomial. Then for the polynomial $h\left(z_{1}\right)$ defined as in (2.16) there exist a polynomial $m\left(z_{1}\right) \in \mathbb{R}\left[z_{1}\right]$ and an odd polynomial $p\left(z_{1}\right)=z_{1} \widehat{p}\left(z_{1}^{2}\right) \in \mathbb{R}\left[z_{1}\right]$ such that

$$
m\left(z_{1}\right) h\left(z_{1}\right)=f\left(z_{1}\right)+z_{1}\left(1-z_{1}\right)^{k_{1}} \widehat{p}\left(z_{1}^{2}\right) .
$$


Proof. Since from P.2 $h\left(z_{1}\right)$ and $z_{1}\left(1-z_{1}\right)^{k_{1}}$ are relatively prime, by using the extended Euclidean algorithm we can find polynomials $\widetilde{m}\left(z_{1}\right)$ and $t\left(z_{1}\right)$ such that

$$
\widetilde{m}\left(z_{1}\right) h\left(z_{1}\right)-z_{1}\left(1-z_{1}\right)^{k_{1}} t\left(z_{1}\right)=f\left(z_{1}\right) .
$$

Further, from Proposition 2.1 and P.1 it follows that the following equation

$$
\rho\left(z_{1}\right) h\left(z_{1}\right)-\rho\left(-z_{1}\right) h\left(-z_{1}\right)=t\left(-z_{1}\right)-t\left(z_{1}\right),
$$

is also solvable. This implies that $\rho\left(z_{1}\right) h\left(z_{1}\right)+t\left(z_{1}\right)$ is an even polynomial, that is,

$$
\rho\left(z_{1}\right) h\left(z_{1}\right)+t\left(z_{1}\right)=\widehat{p}\left(z_{1}^{2}\right) .
$$

Now let us define

$$
m\left(z_{1}\right)=\widetilde{m}\left(z_{1}\right)+z_{1}\left(1-z_{1}\right)^{k_{1}} \rho\left(z_{1}\right) .
$$

We have

$$
\begin{aligned}
m\left(z_{1}\right) h\left(z_{1}\right)-f\left(z_{1}\right) & =\widetilde{m}\left(z_{1}\right) h\left(z_{1}\right)+z_{1}\left(1-z_{1}\right)^{k_{1}} \rho\left(z_{1}\right) h\left(z_{1}\right)-f\left(z_{1}\right) \\
& =z_{1}\left(1-z_{1}\right)^{k_{1}}\left(t\left(z_{1}\right)+\rho\left(z_{1}\right) h\left(z_{1}\right)\right) \\
& =z_{1}\left(1-z_{1}\right)^{k_{1}} \widehat{p}\left(z_{1}^{2}\right) .
\end{aligned}
$$

This theorem can be used to guarantee that the solutions of the systems at step 2 of the proposed construction are indeed Laurent polynomials. Recall that for an invertible square matrix $A$ the adjoint matrix $B=\operatorname{Adj}(A)$ satisfies $B=(\operatorname{det} A) A^{-1}$. If $A$ is a polynomial matrix then $B$ is a polynomial matrix and, therefore, if we require that

$$
f_{j}^{(0)}\left(z_{1}\right)+\left(1-z_{1}\right)^{k_{1}} p_{j}\left(z_{1}\right)=f_{j}^{(0)}\left(z_{1}\right)+z_{1}\left(1-z_{1}\right)^{k_{1}} \widehat{p}_{j}\left(z_{1}^{2}\right)=m_{j}\left(z_{1}\right) h\left(z_{1}\right), \quad 1 \leq j \leq \ell,
$$

then the solution $\boldsymbol{x}^{(j)}$ can be expressed as

$$
\boldsymbol{x}^{(j)}=\frac{m_{j}\left(z_{1}\right)}{z_{1}^{k_{3}\left(k_{3}-1\right) / 2}} \operatorname{Adj}\left(\mathcal{H}^{+}\right) \boldsymbol{e}_{j}, \quad 1 \leq j \leq \ell,
$$

which is a vector whose entries are Laurent polynomials. By incorporating this modification into our strategy we arrive at the following.

\section{The revised idea of the correction polynomial construction}

1. Set $\ell=k_{2}+k_{3}=n$ and $h\left(z_{1}\right)$ as defined in (2.16);

2. Compute the polynomials $p_{j}\left(z_{1}\right)$ and $m_{j}\left(z_{1}\right), 1 \leq j \leq \ell$, satisfying (2.19);

3. Solve $\mathcal{H}^{+} \boldsymbol{x}^{(j)}=\left(f_{j}^{(0)}\left(z_{1}\right)+\left(1-z_{1}\right)^{k_{1}} p_{j}\left(z_{1}\right)\right) \boldsymbol{e}_{j}=h\left(z_{1}\right) m_{j}\left(z_{1}\right) \boldsymbol{e}_{j}, 1 \leq j \leq \ell$;

4. Define the polynomials $x_{j}\left(z_{2}\right)=\sum_{i=0}^{n-1} x_{i}^{(j)} z_{2}^{i}, 1 \leq j \leq \ell$;

5. Set $m\left(z_{1}, z_{2}\right)=\sum_{j=1}^{\ell} x_{j}\left(z_{2}\right)$. 
In light of Remark 2.2 we easily see that the described construction defines a correction $m\left(z_{1}, z_{2}\right)$ satisfying the necessary condition $m(1,1)=1$ as formalized in the following

Proposition 2.7. If $\sum_{j=1}^{\ell} f_{j}^{(0)}(1)=2^{k_{2}+k_{3}}$ and $k_{1}, k_{2}>1$ then the polynomial $m\left(z_{1}, z_{2}\right)$ is such that $m(1,1)=1$.

Remark 2.8. It is important to remark that the degree of polynomial reproduction of the interpolatory subdivision scheme associated with a three-directional box spline obtained by the algebraic strategy just described, is at least the degree of polynomial generation of the box spline subdivision scheme it is built upon. This result simply follows from the algebraic construction of the interpolatory scheme and from the Leibniz rule for the derivatives of a product of symbols (see, for example, [4]).

Remark 2.9. From a computational point of view the solution of the linear systems at step 3 raises several interesting issues. The approach considered above is based on the multiplication of the inverse matrix by the known vector. On the contrary, the typical numerical strategy employs recursive factorization techniques. An alternative recursive method exploiting the factorization properties of Hurwitz matrices is described in Section 4. Furthermore, the case where $f_{j}^{(0)}\left(z_{1}\right)=0,1 \leq j \leq \ell$, providing the so-called "kernel" solutions deserves special attention. Although both solution methods -recursive and inverse-based-can be used, a complete description of the kernel of the associated Hurwitz matrices requires more sophisticated tools like the Smith normal form of polynomial matrices [13]. Applications of this form are presented in the next section.

In the next section we discuss the properties of some interpolatory schemes generated by means of this procedure.

\section{Application examples}

This section is devoted to the application of the described algebraic procedure to four different threedirectional box spline symbols. In particular, we are going to derive the polynomial corrections needed to construct the interpolatory symbols corresponding to the $B_{111}, B_{221}, B_{222}$ (and $B_{331}$ ) box splines. For the corresponding interpolatory subdivision schemes a convergence and smoothness analysis is also conducted together with a polynomial reproduction investigation. For all related theoretical results and definitions we refer the reader to [4] (multivariate extension of the results contained in the papers [8], [9]).

The convergence and smoothness analysis of the considered novel schemes follows here from some results in [10] exploiting functional properties of the associated symbols. Alternative approaches based on a linear algebra technology exploiting the properties of the eigen-decomposition of certain matrices directly constructed from the associated masks have been proposed in [19]. Although these techniques are useful to enlighten several properties of the considered schemes, our preference here is motivated by the intent to focus on the polynomial framework.

\subsection{Interpolatory subdivision schemes associated with the Courant hat function}

We start with the simplest three-directional box spline symbol (the so-called Courant hat function)

$$
a\left(z_{1}, z_{2}\right)=\left(1+z_{1}\right)\left(1+z_{2}\right) \frac{1+z_{1} z_{2}}{2},
$$

which is already interpolatory, though with an associated basic limit function which is only continuous. Therefore, the described construction is used to obtain an interpolatory scheme with increased regularity. In particular, we look for a polynomial correction $m\left(z_{1}, z_{2}\right)$ such that the modified symbol 
$a\left(z_{1}, z_{2}\right) m\left(z_{1}, z_{2}\right)$ fulfills the interpolatory condition (2.4) with $n_{1}=n_{2}=2$. It is straightforward to see that for $c(z)=(1+z)^{2}$, the general solution $(2.11)$ can be expressed as

$$
g\left(z_{1}, z_{2}\right)=z_{2}^{2} p_{0}\left(z_{1}\right)+\left(1-z_{1}\right) t\left(z_{1}, z_{2}\right)=2 z_{1} z_{2}^{2}+\left(1-z_{1}\right) t\left(z_{1}, z_{2}\right),
$$

for a suitable bivariate polynomial $t\left(z_{1}, z_{2}\right)$ which is odd with respect to $z_{1}$. Since $\ell=1+1=2$, we are now going to determine a bivariate polynomial $m\left(z_{1}, z_{2}\right)$ of the form

$$
m\left(z_{1}, z_{2}\right)=\sum_{j=0}^{1} m_{j}\left(z_{1}\right) z_{2}^{j},
$$

satisfying (2.14) for

$$
f_{1}^{(0)}\left(z_{1}\right)=0, \quad f_{2}^{(0)}\left(z_{1}\right)=2 z_{1} .
$$

The coefficients of the polynomial $c\left(z_{2}\right)=\left(1+z_{2}\right) \frac{1+z_{1} z_{2}}{2}$ are used to generate the Hurwitz matrix

$$
\mathcal{H}^{+}=\left[\begin{array}{cc}
1 / 2 & 0 \\
z_{1} / 2 & 1 / 2+1 / 2 z_{1}
\end{array}\right]
$$

whose determinant is $h\left(z_{1}\right)=4^{-1}\left(1+z_{1}\right)$ in accordance with Corollary 2.5 . It is easily observed that

$$
\mathcal{H}^{+}\left[\begin{array}{c}
0 \\
2 z_{1}
\end{array}\right]=\left[\begin{array}{c}
0 \\
2 z_{1}
\end{array}\right]+\left[\begin{array}{c}
0 \\
-z_{1}\left(1-z_{1}\right)
\end{array}\right]
$$

which gives $m\left(z_{1}, z_{2}\right)=z_{1} z_{2}$.

Some other interesting solutions can be obtained by linearly combining $m\left(z_{1}, z_{2}\right)$ with bivariate polynomials satisfying $(2.14)$ with $f_{j}^{(0)}\left(z_{1}\right)=0,1 \leq j \leq \ell$. A simple modification is obtained by considering an admissible known vector of the form

$$
\boldsymbol{f}=\left[z_{1}\left(1-z_{1}\right)\left\{\left(1+z_{1}\right)\left(1-z_{1}\right) p_{0}\left(z_{1}\right)\right\}, z_{1}\left(1-z_{1}\right)\left\{\left(1+z_{1}\right)\left(1-z_{1}\right) p_{1}\left(z_{1}\right)\right\}\right]^{T},
$$

where $p_{0}\left(z_{1}\right)$ and $p_{1}\left(z_{1}\right)$ are suitable even polynomials. Then it follows that the solution of the linear system $\mathcal{H}^{+} \boldsymbol{x}=\boldsymbol{f}$ satisfies

$$
\begin{aligned}
\boldsymbol{x} & =\left(\left(1+z_{1}\right)\left(\mathcal{H}^{+}\right)^{-1}\right)\left[z_{1}\left(1-z_{1}\right)^{2} p_{0}\left(z_{1}\right), z_{1}\left(1-z_{1}\right)^{2} p_{1}\left(z_{1}\right)\right]^{T} \\
& =4 \operatorname{Adj}\left(\mathcal{H}^{+}\right)\left[z_{1}\left(1-z_{1}\right)^{2} p_{0}\left(z_{1}\right), z_{1}\left(1-z_{1}\right)^{2} p_{1}\left(z_{1}\right)\right]^{T} \\
& =2 z_{1}\left(1-z_{1}\right)^{2}\left[\left(1+z_{1}\right) p_{0}\left(z_{1}\right), p_{1}\left(z_{1}\right)-z_{1} p_{0}\left(z_{1}\right)\right] .
\end{aligned}
$$

This gives a parameterized family of symbols defined by

$$
\hat{a}_{\omega}\left(z_{1}, z_{2}\right)=a\left(z_{1}, z_{2}\right)\left\{z_{1} z_{2}+\omega 2 z_{1}\left(1-z_{1}\right)^{2}\left[\left(1+z_{1}\right) p_{0}\left(z_{1}\right)+z_{2}\left(p_{1}\left(z_{1}\right)-z_{1} p_{0}\left(z_{1}\right)\right)\right]\right\} .
$$

More general modifications can be obtained by computing the Smith normal form [13] of a Hurwitz matrix. For instance, let us consider the Hurwitz matrix $\mathcal{H}^{+} \in \mathbb{F}^{6 \times 6}$ generated by the coefficients of the polynomial $c\left(z_{2}\right)=\left(1+z_{2}\right) \frac{1+z_{1} z_{2}}{2}$, namely

$$
\mathcal{H}^{+}=2^{-1}\left[\begin{array}{cccccc}
1 & 0 & 0 & 0 & 0 & 0 \\
z_{1} & 1+z_{1} & 1 & 0 & 0 & 0 \\
0 & 0 & z_{1} & 1+z_{1} & 1 & 0 \\
0 & 0 & 0 & 0 & z_{1} & 1+z_{1} \\
0 & 0 & 0 & 0 & 0 & 0 \\
0 & 0 & 0 & 0 & 0 & 0
\end{array}\right]
$$


Then by computing the Smith normal form of $\mathcal{H}^{+}$we get that

$$
U \mathcal{H}^{+} V=\mathcal{D},
$$

where $U$ and $V$ are unimodular matrices given by

$$
\begin{gathered}
U=\left[\begin{array}{cccccc}
2 & 0 & 0 & 0 & 0 & 0 \\
-2 z_{1} & 2 & 0 & 0 & 0 & 0 \\
2 z_{1}^{2} & -2 z_{1} & 2 & 0 & 0 & 0 \\
-2 z_{1}^{3} & 2 z_{1}^{2} & -2 z_{1} & 2 & 0 & 0 \\
0 & 0 & 0 & 0 & 1 & 0 \\
0 & 0 & 0 & 0 & 0 & 1
\end{array}\right] \\
V=\left[\begin{array}{cccccc}
1 & 0 & 0 & 0 & 0 & 0 \\
0 & 0 & 0 & 0 & 1 & 0 \\
0 & 1 & 0 & 0 & -1-z_{1} & 0 \\
0 & 0 & 0 & 0 & 0 & 1 \\
0 & 0 & 1 & 0 & z_{1}+z_{1}^{2} & -1-z_{1} \\
0 & 0 & 0 & 1 & -z_{1}^{2} & z_{1}
\end{array}\right]
\end{gathered}
$$

and $\mathcal{D}$ is a diagonal matrix defined by

$$
\mathcal{D}=\operatorname{diag}\left[1,1,1,1+z_{1}, 0,0\right] .
$$

In this way we obtain that the general solution of

$$
\mathcal{H}^{+} \boldsymbol{x}=\boldsymbol{f}=\left[0,\left(1-z_{1}\right)^{2}\left(1+z_{1}\right) z_{1} p_{0}\left(z_{1}\right), 0,\left(1-z_{1}\right)^{2}\left(1+z_{1}\right) z_{1} p_{1}\left(z_{1}\right), 0,0\right]^{T},
$$

for suitable even polynomials $p_{0}\left(z_{1}\right)$ and $p_{1}\left(z_{1}\right)$ can be determined as

$$
\boldsymbol{x}=V\left\{\operatorname{diag}\left[1,1,1,\left(1+z_{1}\right)^{-1}, 0,0\right] U \boldsymbol{f}+q_{0}\left(z_{1}\right) \boldsymbol{e}_{5}+q_{1}\left(z_{1}\right) \boldsymbol{e}_{6}\right\},
$$

where $q_{0}\left(z_{1}\right)$ and $q_{1}\left(z_{1}\right)$ are any polynomials. This means that $m_{1}\left(z_{1}, z_{2}\right)=x_{1}+x_{2} z_{2}+x_{3} z_{2}^{2}+x_{4} z_{2}^{3}+$ $x_{5} z_{2}^{4}+x_{6} z_{2}^{5}$ satisfies $(2.14)$ with $f_{j}^{(0)}\left(z_{1}\right)=0,1 \leq j \leq 6$. If $p_{0}\left(z_{1}\right)=1$ and $p_{1}\left(z_{1}\right)=z_{1}^{2}$, for a certain choice of $q_{0}\left(z_{1}\right)$ and $q_{1}\left(z_{1}\right)$, the symbol

$$
\hat{a}_{\omega}\left(z_{1}, z_{2}\right)=a\left(z_{1}, z_{2}\right)\left\{z_{1} z_{2}+\omega m_{1}\left(z_{1}, z_{2}\right)\right\}
$$

defines the Butterfly scheme on the three-directional grid [11] which is $C^{1}$ for $\omega \in(0,0.08)$. In general, the convergence of the subdivision scheme with symbol $\hat{a}_{\omega}\left(z_{1}, z_{2}\right)$ depends on the specific properties of $\omega$ and $m_{1}\left(z_{1}, z_{2}\right)$. Nevertheless, in view of Remark 2.8, whenever the scheme is $C^{0}$, it certainly reproduces at least linear polynomials.

\subsection{Interpolatory subdivision schemes associated with the $B_{221}$ box spline}

In this second example we consider the non-interpolatory box spline symbol defined by

$$
a\left(z_{1}, z_{2}\right)=2^{-2}\left(1+z_{1}\right)^{2}\left(1+z_{2}\right)^{2} \frac{1+z_{1} z_{2}}{2},
$$

and we look for a polynomial correction $m\left(z_{1}, z_{2}\right)$ such that the modified symbol fulfills the interpolatory condition (2.4) with $n_{1}=n_{2}=2$. Since for $c(z)=(1+z)^{2}$ we have

$$
\mathcal{H}^{+}=\left[\begin{array}{ll}
1 & 0 \\
1 & 2
\end{array}\right]
$$


we find that

$$
\left(\mathcal{H}^{+}\right)^{-1}=\left[\begin{array}{cc}
1 & 0 \\
-1 / 2 & 1 / 2
\end{array}\right]
$$

and, therefore, for $n_{1}=2$ the general solution (2.11) can be expressed as

$$
g\left(z_{1}, z_{2}\right)=z_{2}^{2} p_{0}\left(z_{1}\right)+\left(1-z_{1}\right)^{2} t\left(z_{1}, z_{2}\right)=4 z_{1} z_{2}^{2}+\left(1-z_{1}\right)^{2} t\left(z_{1}, z_{2}\right),
$$

for a suitable bivariate polynomial $t\left(z_{1}, z_{2}\right)$ which is odd with respect to $z_{1}$. Since $\ell=2+1=3$, we are now going to determine a bivariate polynomial $m\left(z_{1}, z_{2}\right)$ of the form

$$
m\left(z_{1}, z_{2}\right)=\sum_{j=0}^{2} m_{j}\left(z_{1}\right) z_{2}^{j}
$$

satisfying (2.14) for

$$
f_{1}^{(0)}\left(z_{1}\right)=0, \quad f_{2}^{(0)}\left(z_{1}\right)=4 z_{1}, \quad f_{3}^{(0)}\left(z_{1}\right)=0 .
$$

The coefficients of the polynomial $c\left(z_{2}\right)=\left(1+z_{2}\right)^{2} \frac{1+z_{1} z_{2}}{2}$ are used to generate the Hurwitz matrix

$$
\mathcal{H}^{+}=\left[\begin{array}{ccc}
1 / 2 & 0 & 0 \\
1 / 2+z_{1} & 1+z_{1} / 2 & 1 / 2 \\
0 & z_{1} / 2 & 1 / 2+z_{1}
\end{array}\right]
$$

whose determinant is $h\left(z_{1}\right)=4^{-1}\left(1+z_{1}\right)^{2}$ in accordance with Corollary 2.5 (i.e., $k_{3}\left(k_{3}-1\right)=0$ and $\left.k_{2} k_{3}=2\right)$. For the solution of (2.19) we find that

$$
4 z_{1}=4\left(1+z_{1}\right)^{2} \frac{z_{1}\left(2-z_{1}\right)}{4}-z_{1}\left(1-z_{1}\right)^{2}\left(-2-z_{1}\right),
$$

which gives

$$
\widetilde{m}\left(z_{1}\right)=4 z_{1}\left(2-z_{1}\right), \quad t\left(z_{1}\right)=-2-z_{1} .
$$

Then we have that $\rho\left(z_{1}\right)=2$ fulfills (2.18) and, therefore, we get

$$
m\left(z_{1}\right)=4 z_{1}\left(2-z_{1}\right)+2 z_{1}\left(1-z_{1}\right)^{2}=2 z_{1}\left(5-4 z_{1}+z_{1}^{2}\right) .
$$

Hence, we obtain

$$
\boldsymbol{x}^{(2)}=m\left(z_{1}\right) \operatorname{Adj}\left(\mathcal{H}^{+}\right) \boldsymbol{e}_{2},
$$

which gives

$$
\boldsymbol{x}^{(2)}=m\left(z_{1}\right) \frac{1}{4}\left[\begin{array}{ccc}
2\left(1+z_{1}\right)^{2} & 0 & 0 \\
-\left(1+2 z_{1}\right)^{2} & 1+2 z_{1} & -1 \\
z_{1}\left(1+2 z_{1}\right) & -z_{1} & 2+z_{1}
\end{array}\right] \boldsymbol{e}_{2} .
$$

In this way we arrive at

$$
m\left(z_{1}, z_{2}\right)=\frac{z_{1}\left(5-4 z_{1}+z_{1}^{2}\right)}{4}\left\{\left(1+2 z_{1}\right) z_{2}-z_{1} z_{2}^{2}\right\} .
$$

It can be easily verified that

$$
\hat{a}\left(z_{1}, z_{2}\right)=a\left(z_{1}, z_{2}\right) m\left(z_{1}, z_{2}\right),
$$

is such that

$$
\hat{a}\left(z_{1}, z_{2}\right)+\hat{a}\left(-z_{1}, z_{2}\right)+\hat{a}\left(z_{1},-z_{2}\right)+\hat{a}\left(-z_{1},-z_{2}\right)=8 z_{1}^{2} z_{2}^{2} .
$$

As described in the previous example the properties of the computed solution can be improved by performing a linear combination with some polynomials in the "kernel" of (2.14). In this case a simpler approach comes by reducing the coefficients of $m\left(z_{1}, z_{2}\right)$ modulo $\left(1-z_{1}\right)^{2}$. From

$$
\left(5-4 z_{1}+z_{1}^{2}\right)\left(1+2 z_{1}\right)=\left(-3+2 z_{1}\right)\left(1-z_{1}\right)^{2}+\left(8-2 z_{1}\right),
$$


and

$$
-z_{1}\left(5-4 z_{1}+z_{1}^{2}\right)=\left(2-z_{1}\right)\left(1-z_{1}\right)^{2}-2
$$

we deduce that

$$
m\left(z_{1}, z_{2}\right)=z_{1} z_{2}\left(4-z_{1}-z_{2}\right)+m_{1}\left(z_{1}, z_{2}\right)
$$

where

$$
m_{1}\left(z_{1}, z_{2}\right)=z_{1} z_{2}\left(1-z_{1}\right)^{2}\left\{\left(-3+2 z_{1}\right)+\left(2-z_{1}\right) z_{2}\right\}
$$

It is straightforward to verify that $m_{1}\left(z_{1}, z_{2}\right)$ satisfies $(2.14)$ with $f_{j}^{(0)}\left(z_{1}\right)=0,1 \leq j \leq 3$, and, hence, we conclude that

$$
m_{2}\left(z_{1}, z_{2}\right)=\frac{1}{2} z_{1} z_{2}\left(4-z_{1}-z_{2}\right),
$$

also defines a suitable correction so that $\hat{a}\left(z_{1}, z_{2}\right)=a\left(z_{1}, z_{2}\right) m_{2}\left(z_{1}, z_{2}\right)$ fulfills

$$
\hat{a}\left(z_{1}, z_{2}\right)+\hat{a}\left(-z_{1}, z_{2}\right)+\hat{a}\left(z_{1},-z_{2}\right)+\hat{a}\left(-z_{1},-z_{2}\right)=4 z_{1}^{2} z_{2}^{2} .
$$

Similarly, by employing the shifted solution $p_{0}\left(z_{1}\right)=z_{1}\left(2 z_{1}-1\right)$ it is found that the polynomial

$$
m_{3}\left(z_{1}, z_{2}\right)=\frac{1}{2} z_{1} z_{2}\left(4 z_{1} z_{2}-z_{1}-z_{2}\right),
$$

modifies the symbol in such a way to satisfy the interpolatory condition (2.4) with $n_{1}=n_{2}=4$. As noticed in [6] (though in the univariate setting), any affine combination of the corrections $m_{2}\left(z_{1}, z_{2}\right)$ and $m_{3}\left(z_{1}, z_{2}\right)$ suitably shifted, also generates an interpolatory scheme. In particular the symbol

$$
\begin{aligned}
\hat{a}\left(z_{1}, z_{2}\right) & =\frac{1}{4} a\left(z_{1}, z_{2}\right)\left(m_{2}\left(z_{1}, z_{2}\right)+z_{1}^{-2} z_{2}^{-2} m_{3}\left(z_{1}, z_{2}\right)\right) \\
& =\frac{1}{4} a\left(z_{1}, z_{2}\right)\left(4-z_{1}^{-1}-z_{2}^{-1}+4 z_{1} z_{2}-z_{1}^{2} z_{2}-z_{1} z_{2}^{2}\right),
\end{aligned}
$$

is such that

$$
\hat{a}\left(z_{1}, z_{2}\right)+\hat{a}\left(-z_{1}, z_{2}\right)+\hat{a}\left(z_{1},-z_{2}\right)+\hat{a}\left(-z_{1},-z_{2}\right)=4 z_{1}^{2} z_{2}^{2} \quad \text { and } \quad \hat{a}\left(z_{1}, z_{2}\right)=\hat{a}\left(z_{2}, z_{1}\right) .
$$

The mask of the new interpolatory subdivision scheme is

$$
\hat{\mathbf{a}}=\frac{1}{32}\left(\begin{array}{ccccccc}
0 & 0 & 0 & -1 & -2 & -1 & 0 \\
0 & 0 & -1 & 0 & 2 & 0 & -1 \\
0 & -1 & 4 & 17 & 16 & 2 & -2 \\
-1 & 0 & 17 & 32 & 17 & 0 & -1 \\
-2 & 2 & 16 & 17 & 4 & -1 & 0 \\
-1 & 0 & 2 & 0 & -1 & 0 & 0 \\
0 & -1 & -2 & -1 & 0 & 0 & 0
\end{array}\right)
$$

and the corresponding basic limit function is displayed in Figure 1.

Remark 3.1. It is worthwhile to observe that $m_{2}\left(z_{1}, z_{2}\right)$ in (3.1) is the only solution with symmetric coefficients and degrees (in each variable) less than or equal to 2 , a fact that can be proved via the Smith form. Our computational experience with many other symbols of the form $B_{k_{1}, k_{1}, k_{3}}$ indicates that a similar property holds more generally. For instance, in the case $B_{3,3,1}$ after reduction our procedure generates the correction $m\left(z_{1}, z_{2}\right)$ given by

$$
m\left(z_{1}, z_{2}\right)=\frac{1}{8} z_{1} z_{2}\left(2-9 z_{2}+3 z_{2}^{2}+z_{1}\left(-9+34 z_{2}-9 z_{2}^{2}\right)+z_{1}^{2}\left(3-9 z_{2}+2 z_{2}^{2}\right)\right),
$$

which is the only solution with symmetric coefficients and degrees (in each variable) less than or equal to $\ell-1=3+1-1=3$. This property extends the uniqueness results in [19] for symbols of the form $B_{k, k, k}$. A theoretical proof of the property as well as the analysis of the associated interpolatory schemes is an ongoing work. 


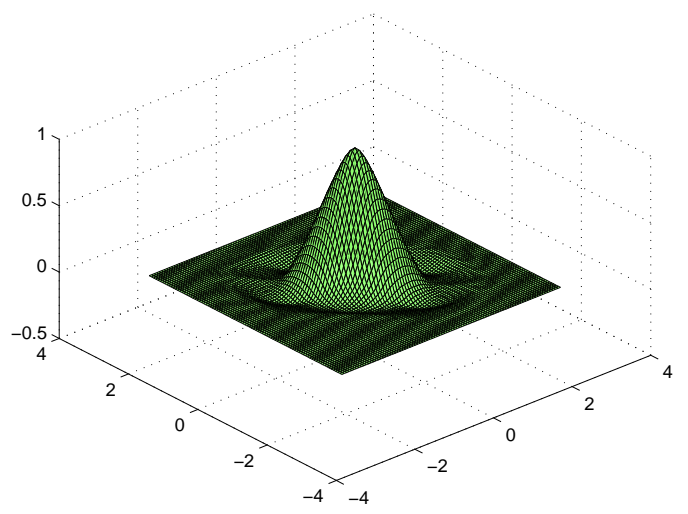

Figure 1: Graph of the basic limit function of the subdivision scheme with mask in (3.3).

\subsubsection{Analysis of the symmetric scheme}

The interpolatory subdivision scheme with symbol (3.2) is symmetric, $C^{1}$ and reproduces polynomials up to degree 2. For its smoothness analysis we make use of [10, Theorem 4.26] exploiting the existence of the three-directional factors $\left(1+z_{1}\right)^{2}\left(1+z_{2}\right)^{2}\left(1+z_{1} z_{2}\right)$ in the subdivision symbol.

Proposition 3.2. The interpolatory subdivision scheme with symbol

$$
\hat{a}\left(z_{1}, z_{2}\right)=\frac{1}{32}\left(1+z_{1}\right)^{2}\left(1+z_{2}\right)^{2}\left(1+z_{1} z_{2}\right)\left(4-z_{1}^{-1}-z_{2}^{-1}+4 z_{1} z_{2}-z_{1}^{2} z_{2}-z_{1} z_{2}^{2}\right),
$$

is $C^{1}$ and reproduces polynomials in $\Pi_{2}$.

Proof. Following [10, Section 4], to prove that the scheme with symbol $\hat{a}\left(z_{1}, z_{2}\right)$ is $C^{1}$ we show that any two of the schemes with symbols

$$
2 \frac{\hat{a}\left(z_{1}, z_{2}\right)}{\left(1+z_{1}\right)}, \quad 2 \frac{\hat{a}\left(z_{1}, z_{2}\right)}{\left(1+z_{2}\right)}, \quad 2 \frac{\hat{a}\left(z_{1}, z_{2}\right)}{\left(1+z_{1} z_{2}\right)},
$$

are $C^{0}$. In particular we consider the first and the second symbols above, that is

$$
\frac{1}{16}\left(1+z_{1}\right)\left(1+z_{2}\right)^{2}\left(1+z_{1} z_{2}\right)\left(4-z_{1}^{-1}-z_{2}^{-1}+4 z_{1} z_{2}-z_{1}^{2} z_{2}-z_{1} z_{2}^{2}\right)
$$

and

$$
\frac{1}{16}\left(1+z_{1}\right)^{2}\left(1+z_{2}\right)\left(1+z_{1} z_{2}\right)\left(4-z_{1}^{-1}-z_{2}^{-1}+4 z_{1} z_{2}-z_{1}^{2} z_{2}-z_{1} z_{2}^{2}\right),
$$

and check that their difference schemes are contractive. To this purpose, for

$$
b_{1}\left(z_{1}, z_{2}\right)=\frac{1}{16}\left(1+z_{1}\right)\left(1+z_{2}\right)\left(1+z_{1} z_{2}\right)\left(4-z_{1}^{-1}-z_{2}^{-1}+4 z_{1} z_{2}-z_{1}^{2} z_{2}-z_{1} z_{2}^{2}\right),
$$

and

$$
\begin{aligned}
& b_{2}\left(z_{1}, z_{2}\right)=\frac{1}{16}\left(1+z_{1}\right)^{2}\left(1+z_{2}\right)\left(4-z_{1}^{-1}-z_{2}^{-1}+4 z_{1} z_{2}-z_{1}^{2} z_{2}-z_{1} z_{2}^{2}\right), \\
& b_{3}\left(z_{1}, z_{2}\right)=\frac{1}{16}\left(1+z_{1}\right)\left(1+z_{2}\right)^{2}\left(4-z_{1}^{-1}-z_{2}^{-1}+4 z_{1} z_{2}-z_{1}^{2} z_{2}-z_{1} z_{2}^{2}\right),
\end{aligned}
$$

we verify that $b_{i}\left(z_{1}, z_{2}\right) b_{i}\left(z_{1}^{2}, z_{2}^{2}\right), i=1,2,3$ are indeed contractive.

The polynomial reproduction properties of the scheme follow from the polynomial generation properties of the box spline subdivision scheme $a\left(z_{1}, z_{2}\right)=\frac{1}{8}\left(1+z_{1}\right)^{2}\left(1+z_{2}\right)^{2}\left(1+z_{1} z_{2}\right)$. 
The properties of the new interpolatory subdivision scheme have been analyzed by relying on the results in [10]. Obviously, the same conclusions can be derived regardless the approach taken in the analysis. As a matter of fact, we have also generated the $49 \times 49$ matrix $H$ determined by the mask elements (that is the $7 \times 7$ block matrix with blocks $H_{k, \ell}, k, \ell=1, \ldots, 7$ defined as $H_{k, \ell}(i, j)=$ $\hat{a}(2 j-i, 2 \ell-k), i, j=1, \ldots, 7)$. It is easily found that this matrix has 1 as a simple eigenvalue with $(1, \cdots, 1)^{T}$ as its eigenvector. Hence, from [19, Theorem 1.2] we can conclude the existence of a function refinable with respect to the given mask, which is a fundamental solution for cardinal interpolation.

\subsection{Interpolatory subdivision schemes associated with the $B_{222}$ box spline}

As third application example we consider the approximating box spline symbol defined by

$$
a\left(z_{1}, z_{2}\right)=2^{-2}\left(1+z_{1}\right)^{2}\left(1+z_{2}\right)^{2}\left(\frac{1+z_{1} z_{2}}{2}\right)^{2} .
$$

Again, we look for a polynomial correction $m\left(z_{1}, z_{2}\right)$ such that the modified symbol fulfills the interpolatory condition (2.4) with $n_{1}=n_{2}=4$. It is found that the general solution (2.11) can be expressed as

$$
g\left(z_{1}, z_{2}\right)=z_{2}^{4} p_{0}\left(z_{1}\right)+\left(1-z_{1}\right)^{2} t\left(z_{1}, z_{2}\right)
$$

where

$$
p_{0}\left(z_{1}\right)=z_{1}\left(-1+2 z_{1}+31 z_{1}^{2}\right)
$$

and $t\left(z_{1}, z_{2}\right)$ is a suitable bivariate polynomial which is odd with respect to $z_{1}$. Then, by using the same approach described in the previous example, after some simplifications, the correction $m\left(z_{1}, z_{2}\right)$ given by

$$
m\left(z_{1}, z_{2}\right)=-\frac{z_{1} z_{2}}{4}\left(1+z_{1}+z_{2}-10 z_{1} z_{2}+z_{1}^{2} z_{2}+z_{1} z_{2}^{2}+z_{1}^{2} z_{2}^{2}\right)
$$

is computed. The mask of the new interpolatory subdivision scheme $\hat{a}\left(z_{1}, z_{2}\right)=m\left(z_{1}, z_{2}\right) a\left(z_{1}, z_{2}\right)$ is

$$
\hat{\mathbf{a}}=\frac{1}{64}\left(\begin{array}{ccccccc}
0 & 0 & 0 & -1 & -3 & -3 & -1 \\
0 & 0 & -3 & 0 & 6 & 0 & -3 \\
0 & -3 & 6 & 33 & 33 & 6 & -3 \\
-1 & 0 & 33 & 64 & 33 & 0 & -1 \\
-3 & 6 & 33 & 33 & 6 & -3 & 0 \\
-3 & 0 & 6 & 0 & -3 & 0 & 0 \\
-1 & -3 & -3 & -1 & 0 & 0 & 0
\end{array}\right)
$$

Note that the interpolatory mask in (3.5) is the one obtained for $B_{222}$ in [19].

\subsubsection{Analysis of the new interpolatory subdivision scheme}

Also in this case, the algebraic construction leads to a new interesting interpolatory bivariate subdivision scheme: the interpolatory scheme with correction (3.4) is symmetric, $C^{1}$ and reproducing $\Pi_{3}$.

Proposition 3.3. The interpolatory subdivision scheme with symbol

$$
\hat{a}\left(z_{1}, z_{2}\right)=\frac{1}{64} z_{1} z_{2}\left(1+z_{1}\right)^{2}\left(1+z_{2}\right)^{2}\left(1+z_{1} z_{2}\right)^{2}\left(10 z_{1} z_{2}-1-z_{1}-z_{2}-z_{1}^{2} z_{2}-z_{1} z_{2}^{2}-z_{1}^{2} z_{2}^{2}\right),
$$

is $C^{1}$ and reproduces polynomials in $\Pi_{3}$. 


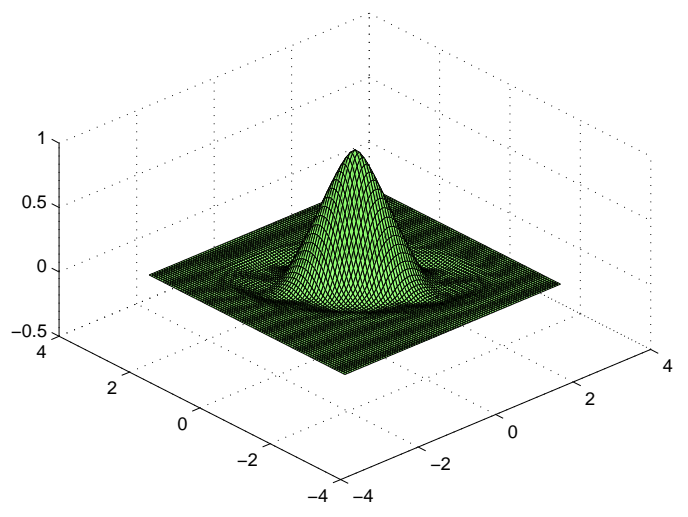

Figure 2: Graph of the basic limit function of the subdivision scheme with mask in (3.5).

Proof. Following [10, Section 4], to prove that the scheme with symbol $\hat{a}\left(z_{1}, z_{2}\right)$ is $C^{1}$ we show that any two of the schemes with symbols

$$
2 \frac{\hat{a}\left(z_{1}, z_{2}\right)}{\left(1+z_{1}\right)}, \quad 2 \frac{\hat{a}\left(z_{1}, z_{2}\right)}{\left(1+z_{2}\right)}, \quad 2 \frac{\hat{a}\left(z_{1}, z_{2}\right)}{\left(1+z_{1} z_{2}\right)},
$$

are $C^{0}$. In particular we consider the first and the second symbols above that is

$$
\frac{1}{32} z_{1} z_{2}\left(1+z_{1}\right)\left(1+z_{2}\right)^{2}\left(1+z_{1} z_{2}\right)^{2}\left(10 z_{1} z_{2}-1-z_{1}-z_{2}-z_{1}^{2} z_{2}-z_{1} z_{2}^{2}-z_{1}^{2} z_{2}^{2}\right),
$$

and

$$
\frac{1}{32} z_{1} z_{2}\left(1+z_{1}\right)^{2}\left(1+z_{2}\right)\left(1+z_{1} z_{2}\right)^{2}\left(10 z_{1} z_{2}-1-z_{1}-z_{2}-z_{1}^{2} z_{2}-z_{1} z_{2}^{2}-z_{1}^{2} z_{2}^{2}\right) .
$$

For these symbols we check whether their difference symbols

$$
\begin{aligned}
& b_{1}\left(z_{1}, z_{2}\right)=\frac{1}{32} z_{1} z_{2}\left(1+z_{1}\right)\left(1+z_{2}\right)\left(1+z_{1} z_{2}\right)^{2}\left(10 z_{1} z_{2}-1-z_{1}-z_{2}-z_{1}^{2} z_{2}-z_{1} z_{2}^{2}-z_{1}^{2} z_{2}^{2}\right), \\
& b_{2}\left(z_{1}, z_{2}\right)=\frac{1}{32} z_{1} z_{2}\left(1+z_{1}\right)\left(1+z_{2}\right)^{2}\left(1+z_{1} z_{2}\right)\left(10 z_{1} z_{2}-1-z_{1}-z_{2}-z_{1}^{2} z_{2}-z_{1} z_{2}^{2}-z_{1}^{2} z_{2}^{2}\right), \\
& b_{3}\left(z_{1}, z_{2}\right)=\frac{1}{32} z_{1} z_{2}\left(1+z_{1}\right)^{2}\left(1+z_{2}\right)\left(1+z_{1} z_{2}\right)\left(10 z_{1} z_{2}-1-z_{1}-z_{2}-z_{1}^{2} z_{2}-z_{1} z_{2}^{2}-z_{1}^{2} z_{2}^{2}\right),
\end{aligned}
$$

are contractive. Indeed it holds that the norm of $b_{i}\left(z_{1}, z_{2}\right) b_{i}\left(z_{1}^{2}, z_{2}^{2}\right)$, for all $i=1,2,3$ is less than 1 . The polynomial reproduction properties of the scheme follow from the polynomial generation properties of the box spline subdivision scheme $a\left(z_{1}, z_{2}\right)=\frac{1}{64}\left(1+z_{1}\right)^{2}\left(1+z_{2}\right)^{2}\left(1+z_{1} z_{2}\right)^{2}$.

Remark 3.4. We remark that the scheme with mask (3.5), due to the extra smoothing factor $\left(1+z_{1} z_{2}\right)$, is somehow smoother than the one with mask (3.3), even if this does not emerge form the conducted regularity analysis.

\section{A recursive approach for solving Hurwitz systems over integral domains}

The constructions presented in the previous section basically reduce the computation of a polynomial correction $m\left(z_{1}, z_{2}\right)$ to solving some linear systems whose coefficient matrix is a Hurwitz matrix with entries over the integral domain $\mathbb{F}=\mathbb{R}\left[z_{1}\right]$. The computation of the determinant of a polynomial matrix as well as the solution of a linear system can be carried out by using the Hermite normal form of the matrix [18] or some other triangularization procedure working on the integral domain $\mathbb{R}[z]$. In 
the case of Hurwitz and Sylvester matrices it is common to reinterpret matrix factorization methods in the context of a polynomial framework. In this section we provide a polynomial method for solving (2.14) as a supplementary material. The computational interest of the procedure is that it generally leads to a different solution and would make possible to exploit special properties of the symbol.

For the sake of illustration, let us start by continuing the discussion with the example presented in Subsection 3.2. Recall that our task is to compute univariate polynomials $m_{j}\left(z_{1}\right), j=0,1,2$, such that

$$
\left[\begin{array}{ccc}
1 / 2 & 0 & 0 \\
1 / 2+z_{1} & 1+z_{1} / 2 & 1 / 2 \\
0 & z_{1} / 2 & 1 / 2+z_{1}
\end{array}\right]\left[\begin{array}{c}
m_{0}\left(z_{1}\right) \\
m_{1}\left(z_{1}\right) \\
m_{2}\left(z_{1}\right)
\end{array}\right]=\left[\begin{array}{c}
\left(1-z_{1}\right)^{2} z_{1} p_{1}\left(z_{1}^{2}\right) \\
4 z_{1}+\left(1-z_{1}\right)^{2} z_{1} p_{2}\left(z_{1}^{2}\right) \\
\left(1-z_{1}\right)^{2} z_{1} p_{3}\left(z_{1}^{2}\right)
\end{array}\right]
$$

for suitable polynomials $p_{j}\left(z_{1}\right), j=1,2,3$. The first equation can be satisfied by setting $m_{0}\left(z_{1}\right)=0$ and $p_{1}\left(z_{1}\right)=0$. This yields the reduced system

$$
\left[\begin{array}{cc}
1+z_{1} / 2 & 1 / 2 \\
z_{1} / 2 & 1 / 2+z_{1}
\end{array}\right]\left[\begin{array}{c}
m_{1}\left(z_{1}\right) \\
m_{2}\left(z_{1}\right)
\end{array}\right]=\left[\begin{array}{c}
4 z_{1}+\left(1-z_{1}\right)^{2} z_{1} p_{2}\left(z_{1}^{2}\right) \\
\left(1-z_{1}\right)^{2} z_{1} p_{3}\left(z_{1}^{2}\right)
\end{array}\right]
$$

The coefficient matrix on the left hand side can be reduced to a triangular form by multiplication on the right by the triangular matrix

$$
G=\left[\begin{array}{cc}
1 & -1 / 2 \\
0 & 1+z_{1} / 2
\end{array}\right]
$$

We have that

$$
\left[\begin{array}{cc}
1+z_{1} / 2 & 1 / 2 \\
z_{1} / 2 & 1 / 2+z_{1}
\end{array}\right] G=\left[\begin{array}{cc}
1+z_{1} / 2 & 0 \\
z_{1} / 2 & \frac{\left(1+z_{1}\right)^{2}}{2}
\end{array}\right]
$$

Since

$$
\left[\begin{array}{cc}
1 & -1 / 2 \\
0 & 1+z_{1} / 2
\end{array}\right]\left[\begin{array}{cc}
1+z_{1} / 2 & 1 / 2 \\
0 & 1
\end{array}\right]=\left(1+z_{1} / 2\right) I_{2}
$$

then we can rewrite the reduced system in the following form

$$
\left[\begin{array}{cc}
1+z_{1} / 2 & 0 \\
z_{1} / 2 & \frac{\left(1+z_{1}\right)^{2}}{2}
\end{array}\right]\left[\begin{array}{l}
m_{1}^{(1)}\left(z_{1}\right) \\
m_{2}^{(1)}\left(z_{1}\right)
\end{array}\right]=\left(1+z_{1} / 2\right)\left[\begin{array}{c}
4 z_{1}+\left(1-z_{1}\right)^{2} z_{1} p_{2}\left(z_{1}^{2}\right) \\
\left(1-z_{1}\right)^{2} z_{1} p_{3}\left(z_{1}^{2}\right)
\end{array}\right]
$$

where

$$
\left[\begin{array}{l}
m_{1}^{(1)}\left(z_{1}\right) \\
m_{2}^{(1)}\left(z_{1}\right)
\end{array}\right]=\left[\begin{array}{cc}
1+z_{1} / 2 & 1 / 2 \\
0 & 1
\end{array}\right]\left[\begin{array}{l}
m_{1}\left(z_{1}\right) \\
m_{2}\left(z_{1}\right)
\end{array}\right]
$$

We look for a solution of the reduced system where the polynomials $m_{j}^{(1)}\left(z_{1}\right), j=1,2$, are such that $m_{j}^{(1)}\left(z_{1}\right)=\left(1+z_{1} / 2\right) \hat{m}_{j}^{(1)}\left(z_{1}\right), j=1,2$. In this way from the first equation we obtain that

$$
\left(1+z_{1} / 2\right) \hat{m}_{1}^{(1)}\left(z_{1}\right)=4 z_{1}+\left(1-z_{1}\right)^{2} z_{1} p_{2}\left(z_{1}^{2}\right) .
$$

This equation has the form (2.19) and it can be solved by setting

$$
\hat{m}_{1}^{(1)}\left(z_{1}\right)=\frac{8}{9} z_{1}\left(4-z_{1}\right), \quad p_{2}\left(z_{1}\right)=-\frac{4}{9} .
$$

Hence, by replacing $\hat{m}_{1}^{(1)}\left(z_{1}\right)$ into the second equation it is found that

$$
\frac{\left(1+z_{1}\right)^{2}}{2} \hat{m}_{2}^{(1)}\left(z_{1}\right)=\frac{4}{9} z_{1}^{2}\left(z_{1}-4\right)+\left(1-z_{1}\right)^{2} z_{1} p_{3}\left(z_{1}^{2}\right) .
$$

This is again a polynomial equation of the form (2.19) and a solution is given by

$$
\hat{m}_{2}^{(1)}\left(z_{1}\right)=z_{1}\left(-1+\frac{4}{9} z_{1}-\frac{1}{9} z_{1}^{2}\right), \quad p_{3}\left(z_{1}\right)=\frac{1}{2}+\frac{1}{18} z_{1} .
$$


Now we are in position to determine the polynomial coefficients $m_{1}\left(z_{1}\right)$ and $m_{2}\left(z_{1}\right)$. By using (4.1) we deduce that

$$
\left(1+z_{1} / 2\right)\left[\begin{array}{l}
\hat{m}_{1}^{(1)}\left(z_{1}\right) \\
\hat{m}_{2}^{(1)}\left(z_{1}\right)
\end{array}\right]=\left[\begin{array}{cc}
1+z_{1} / 2 & 1 / 2 \\
0 & 1
\end{array}\right]\left[\begin{array}{l}
m_{1}\left(z_{1}\right) \\
m_{2}\left(z_{1}\right)
\end{array}\right] .
$$

Hence, we find that

$$
\left(1+z_{1} / 2\right)\left[\begin{array}{l}
\hat{m}_{1}^{(1)}\left(z_{1}\right) \\
\hat{m}_{2}^{(1)}\left(z_{1}\right)
\end{array}\right]=\left[\begin{array}{cc}
1+z_{1} / 2 & 1 / 2 \\
0 & 1
\end{array}\right]\left[\begin{array}{cc}
1 & -1 / 2 \\
0 & 1+z_{1} / 2
\end{array}\right]\left[\begin{array}{c}
\hat{m}_{1}^{(1)}\left(z_{1}\right) \\
\hat{m}_{2}^{(1)}\left(z_{1}\right)
\end{array}\right]
$$

which gives

$$
\left[\begin{array}{cc}
1 & -1 / 2 \\
0 & 1+z_{1} / 2
\end{array}\right]\left[\begin{array}{c}
\hat{m}_{1}^{(1)}\left(z_{1}\right) \\
\hat{m}_{2}^{(1)}\left(z_{1}\right)
\end{array}\right]=\left[\begin{array}{c}
m_{1}\left(z_{1}\right) \\
m_{2}\left(z_{1}\right)
\end{array}\right]
$$

and, therefore,

$$
\left[\begin{array}{l}
m_{1}\left(z_{1}\right) \\
m_{2}\left(z_{1}\right)
\end{array}\right]=z_{1}\left[\begin{array}{c}
\frac{73}{18}-\frac{10 z_{1}}{9}+\frac{z_{1}^{2}}{18} \\
-1-\frac{z_{1}}{18}+\frac{z_{1}^{2}}{9}-\frac{z_{1}^{3}}{18}
\end{array}\right]
$$

This solution can be simplified by operating modulo $\left(1-z_{1}\right)^{2}$. The remainder of $m_{1}\left(z_{1}\right) / z_{1}$ and $m_{2}\left(z_{1}\right) / z_{1}$ by $\left(1-z_{1}\right)^{2}$ is $4-z_{1}$ and -1 , respectively. Therefore, in this way we arrive at the same solution $m_{2}\left(z_{1}, z_{2}\right)$ determined in Subsection 3.2.

The approach described above for solving (2.14) in the case of the $B_{221}$ box spline symbol can be suitably generalized and reinterpreted in a polynomial framework.

Let $\mathcal{H}_{0}^{+} \in \mathbb{F}^{n \times n}, \mathbb{F}=\mathbb{R}\left[z_{1}\right], n=\ell=k_{2}+k_{3}$, be the Hurwitz matrix generated from the coefficients of $c^{(0)}\left(z_{2}\right)=c\left(z_{2}\right)=\sum_{i=0}^{k_{2}+k_{3}} g_{i}\left(z_{1}\right) z_{2}^{i}$, namely

$$
\mathcal{H}_{0}^{+}:=\left[\begin{array}{ccccc}
g_{0}\left(z_{1}\right) & 0 & \ldots & \ldots & \ldots \\
g_{2}\left(z_{1}\right) & g_{1}\left(z_{1}\right) & g_{0}\left(z_{1}\right) & 0 & \ldots \\
g_{4}\left(z_{1}\right) & g_{3}\left(z_{1}\right) & g_{2}\left(z_{1}\right) & \ldots & \ldots \\
\vdots & \vdots & \vdots & \vdots & \ldots \\
\vdots & \vdots & \vdots & \vdots & \ldots \\
g_{2(n-1)}\left(z_{1}\right) & g_{2(n-1)-1}\left(z_{1}\right) & g_{2(n-1)-2}\left(z_{1}\right) & \ldots & \ldots
\end{array}\right] .
$$

We have to solve a linear system

$$
\mathcal{H}_{0}^{+}\left[\begin{array}{c}
m_{0}^{(0)}\left(z_{1}\right) \\
\vdots \\
m_{n-1}^{(0)}\left(z_{1}\right)
\end{array}\right]=\left[\begin{array}{c}
f_{1}^{(0)}\left(z_{1}\right)+\left(1-z_{1}\right)^{2} z_{1} p_{1}\left(z_{1}^{2}\right) \\
\vdots \\
f_{n}^{(0)}\left(z_{1}\right)+\left(1-z_{1}\right)^{2} z_{1} p_{n}\left(z_{1}^{2}\right)
\end{array}\right]
$$

where $f_{j}^{(0)}\left(z_{1}\right)$ are given and, moreover, $p_{j}\left(z_{1}\right)$ have to be suitably chosen in such a way to admit a polynomial solution. The first step is trying to solve the first equation having the form of (2.19), that is

$$
g_{0}\left(z_{1}\right) m_{0}\left(z_{1}\right)=f_{1}^{(0)}\left(z_{1}\right)+\left(1-z_{1}\right)^{2} z_{1} p_{1}\left(z_{1}^{2}\right) .
$$

If we succeed to do that, then we can reduce the size of the system by obtaining

$$
\mathcal{H}_{0}^{+}(2: n ; 2: n)\left[\begin{array}{c}
m_{1}^{(0)}\left(z_{1}\right) \\
\vdots \\
m_{n-1}^{(0)}\left(z_{1}\right)
\end{array}\right]=\left[\begin{array}{c}
f_{2}^{(1)}\left(z_{1}\right)+\left(1-z_{1}\right)^{2} z_{1} p_{1}\left(z_{1}^{2}\right) \\
\vdots \\
f_{n}^{(1)}\left(z_{1}\right)+\left(1-z_{1}\right)^{2} z_{1} p_{n}\left(z_{1}^{2}\right)
\end{array}\right] .
$$


Let $G^{+} \in \mathbb{F}^{2 \times 2}$ and $G^{-} \in \mathbb{F}^{2 \times 2}$ be the triangular matrices defined by

$$
G^{+}=\left[\begin{array}{cc}
1 & -g_{0}\left(z_{1}\right) \\
0 & g_{1}\left(z_{1}\right)
\end{array}\right], \quad G^{-}=\left[\begin{array}{cc}
g_{1}\left(z_{1}\right) & g_{0}\left(z_{1}\right) \\
0 & 1
\end{array}\right] .
$$

Observe that

$$
G^{+} G^{-}=G^{-} G^{+}=g_{1}\left(z_{1}\right) I_{2} .
$$

According to the parity of $n-1$ we introduce the cumulative elimination matrix $\mathcal{G}^{+} \in \mathbb{F}^{(n-1) \times(n-1)}$ defined as follows: if $n-1$ is even then

$$
\mathcal{G}^{+}=G^{+} \oplus G^{+} \oplus \ldots \oplus G^{+}
$$

otherwise, if $n-1$ is odd

$$
\mathcal{G}^{+}=G^{+} \oplus G^{+} \oplus \ldots \oplus G^{+} \oplus 1 .
$$

Similarly, we define $\mathcal{G}^{-} \in \mathbb{F}^{(n-1) \times(n-1)}$ by setting

$$
\mathcal{G}^{-}=G^{-} \oplus G^{-} \oplus \ldots \oplus G^{-}
$$

or

$$
\mathcal{G}^{-}=G^{-} \oplus G^{-} \oplus \ldots \oplus G^{-} \oplus g_{1}\left(z_{1}\right)
$$

From (4.3) there follows that

$$
\mathcal{G}^{+} \mathcal{G}^{-}=\mathcal{G}^{-} \mathcal{G}^{+}=g_{1}\left(z_{1}\right) I_{n-1}
$$

By using this relation we can rewrite the reduced system into the equivalent form

$$
\mathcal{H}_{0}^{+}(2: n ; 2: n) \mathcal{G}^{+} \mathcal{G}^{-}\left[\begin{array}{c}
m_{1}^{(0)}\left(z_{1}\right) \\
\vdots \\
m_{n-1}^{(0)}\left(z_{1}\right)
\end{array}\right]=g_{1}\left(z_{1}\right)\left[\begin{array}{c}
f_{2}^{(1)}\left(z_{1}\right)+\left(1-z_{1}\right)^{2} z_{1} p_{1}\left(z_{1}^{2}\right) \\
\vdots \\
f_{n}^{(1)}\left(z_{1}\right)+\left(1-z_{1}\right)^{2} z_{1} p_{n}\left(z_{1}^{2}\right)
\end{array}\right] .
$$

The matrix $\mathcal{H}_{1}^{+}=\mathcal{H}_{0}^{+}(2: n ; 2: n) \mathcal{G}^{+}$is the Hurwitz matrix associated with a transformed polynomial $c^{(1)}\left(z_{2}\right)$ generated according to the following rules: given the even-odd decomposition of $c^{(0)}\left(z_{2}\right)$, i.e.,

$$
c^{(0)}\left(z_{2}\right)=c_{e}^{(0)}\left(z_{2}^{2}\right)+z_{2} c_{o}^{(0)}\left(z_{2}^{2}\right),
$$

then we obtain

$$
c^{(1)}\left(z_{2}\right)=c_{o}^{(0)}\left(z_{2}^{2}\right)+z_{2} \frac{c_{o}^{(0)}(0) c_{e}^{(0)}\left(z_{2}^{2}\right)-c_{e}^{(0)}(0) c_{o}^{(0)}\left(z_{2}^{2}\right)}{z_{2}^{2}},
$$

which gives the transformation rules

$$
c_{e}^{(1)}\left(z_{2}\right)=c_{o}^{(0)}\left(z_{2}\right), \quad c_{o}^{(1)}\left(z_{2}\right)=\frac{c_{o}^{(0)}(0) c_{e}^{(0)}\left(z_{2}\right)-c_{e}^{(0)}(0) c_{o}^{(0)}\left(z_{2}\right)}{z_{2}} .
$$

It is worth noting that the degree of $c^{(1)}\left(z_{2}\right)$ is at least 1 minus the degree of $c^{(0)}\left(z_{2}\right)$. The solution of the reduced system of order $n-1$ is denoted by

$$
\left[\begin{array}{c}
m_{1}^{(1)}\left(z_{1}\right) \\
\vdots \\
m_{n-1}^{(1)}\left(z_{1}\right)
\end{array}\right]:=\mathcal{G}^{-}\left[\begin{array}{c}
m_{1}^{(0)}\left(z_{1}\right) \\
\vdots \\
m_{n-1}^{(0)}\left(z_{1}\right)
\end{array}\right]
$$

Suppose that we are looking for polynomials $m_{j}^{(1)}\left(z_{1}\right)$ satisfying $m_{j}^{(1)}\left(z_{1}\right)=g_{1}\left(z_{1}\right) \hat{m}_{j}^{(1)}\left(z_{1}\right), j=$ $1, \ldots, n-1$ and then define $m_{1}\left(z_{1}, z_{2}\right)=\sum_{j=1}^{n-1} \hat{m}_{j}^{(1)}\left(z_{1}\right) z_{2}^{j-1}$. The coefficient of this correction polynomial solves

$$
\mathcal{H}_{1}^{+}\left[\begin{array}{c}
\hat{m}_{1}^{(1)}\left(z_{1}\right) \\
\vdots \\
\hat{m}_{n-1}^{(1)}\left(z_{1}\right)
\end{array}\right]=\left[\begin{array}{c}
f_{2}^{(1)}\left(z_{1}\right)+\left(1-z_{1}\right)^{2} z_{1} p_{1}\left(z_{1}^{2}\right) \\
\vdots \\
f_{n}^{(1)}\left(z_{1}\right)+\left(1-z_{1}\right)^{2} z_{1} p_{n}\left(z_{1}^{2}\right)
\end{array}\right]
$$


which is equivalent in a polynomial setting to the equation of reduced degree

$$
\sum_{j=1}^{n-1}\left(f_{j+1}^{(1)}\left(z_{1}\right)+\left(1-z_{1}\right)^{k_{1}} p_{j}\left(z_{1}\right)\right) z_{2}^{2(j-1)}=c^{(1)}\left(z_{2}\right) m_{1}\left(z_{1}, z_{2}\right)+c^{(1)}\left(-z_{2}\right) m_{1}\left(z_{1},-z_{2}\right) .
$$

The transformation from (2.14) to this equation of reduced degree can be summarized as follows:

\section{The polynomial reduction process}

1. Compute $m_{0}\left(z_{1}\right)$ by solving (4.2);

2. Compute the polynomial $f_{j}^{(1)}\left(z_{1}\right)=f_{j}^{(0)}\left(z_{1}\right)-m_{0}\left(z_{1}\right) g_{2(j-1)}\left(z_{1}\right), 2 \leq j \leq n$;

3. Compute the coefficients of $c^{(1)}\left(z_{2}\right)=\sum_{i=0}^{k_{2}+k_{3}-1} g_{i}^{(1)}\left(z_{1}\right) z_{2}^{i}$ from (4.4).

This process can in principle be continued until we arrive at a polynomial equation in one single variable (i.e., of degree zero). After that, we have to reconstruct the coefficients of the initial correction $m_{0}\left(z_{1}, z_{2}\right)$ starting from the coefficients of the polynomials $m_{j}\left(z_{1}, z_{2}\right)$ generated step-by-step in the degree-reduction procedure. Let us suppose that $m_{1}\left(z_{1}, z_{2}\right)$ is known and we have to proceed with the reconstruction of $m_{0}\left(z_{1}, z_{2}\right)$. From

$$
\mathcal{G}^{-}\left[\begin{array}{c}
m_{1}^{(0)}\left(z_{1}\right) \\
\vdots \\
m_{n-1}^{(0)}\left(z_{1}\right)
\end{array}\right]=\left[\begin{array}{c}
m_{1}^{(1)}\left(z_{1}\right) \\
\vdots \\
m_{n-1}^{(1)}\left(z_{1}\right)
\end{array}\right]=g_{1}\left(z_{1}\right)\left[\begin{array}{c}
\hat{m}_{1}^{(1)}\left(z_{1}\right) \\
\vdots \\
\hat{m}_{n-1}^{(1)}\left(z_{1}\right)
\end{array}\right]=\mathcal{G}^{-} \mathcal{G}^{+}\left[\begin{array}{c}
\hat{m}_{1}^{(1)}\left(z_{1}\right) \\
\vdots \\
\hat{m}_{n-1}^{(1)}\left(z_{1}\right)
\end{array}\right]
$$

we deduce that

$$
\left[\begin{array}{c}
m_{1}^{(0)}\left(z_{1}\right) \\
\vdots \\
m_{n-1}^{(0)}\left(z_{1}\right)
\end{array}\right]=\mathcal{G}^{+}\left[\begin{array}{c}
\hat{m}_{1}^{(1)}\left(z_{1}\right) \\
\vdots \\
\hat{m}_{n-1}^{(1)}\left(z_{1}\right)
\end{array}\right]
$$

which makes possible to compute the coefficients $m_{j}^{(0)}$ of $m\left(z_{1}, z_{2}\right)=m_{0}\left(z_{1}, z_{2}\right)$ starting from the coefficients $\hat{m}_{j}^{(1)}$ of $m_{1}\left(z_{1}, z_{2}\right)$ by performing polynomial multiplications only.

\section{Conclusions and future work}

In this paper we devise a general algebraic strategy for the construction of interpolatory bivariate subdivision schemes induced by the three-directional box spline symbols. The proposed approach is able to generate large families of interpolatory schemes depending on the algebraic properties of the Hurwitz matrices associated with the symbols. Future work includes the systematic analysis of these schemes as well as the characterization of the properties of the Hurwitz matrices in terms of the properties of the related box spline symbols. Also, it is our intention to extend these results to the case where a symbol factor is not of box spline type.

\section{References}

[1] S. Basu, R. Pollack, M. F. Roy, Algorithms in real algebraic geometry, Algorithms and Computation in Mathematics 10, Springer-Verlag, 2006. 
[2] A. S. Cavaretta, W. Dahmen, and C. A. Micchelli, Stationary Subdivision, Mem. Amer. Math. Soc., 453 (1991).

[3] M. Charina, C. Conti and T. Sauer, Regularity of Multivariate Vector Subdivision Schemes, Numerical Algorithms, 39, 97-113 (2005).

[4] M. Charina and C. Conti, Polynomial reproduction of multivariate scalar subdivision schemes, Journal of Computational and Applied Mathematics (2012), doi:10.1016/j.cam.2012.06.013

[5] M. Charina, C. Conti, K. Jetter and G. Zimmermann, Scalar multivariate subdivision schemes and box splines, Computer Aided Geometric Design, Volume 28, Issue 5, 285-306 (2011).

[6] C. Conti, L. Gemignani, L. Romani, From symmetric subdivision masks of Hurwitz type to interpolatory subdivision masks. Linear Algebra and its Applications, 431, 1971-1987 (2009).

[7] C. Conti, L. Gemignani, L. Romani, From approximating to interpolatory non-stationary subdivision schemes with the same generation properties, Advances in Computational Mathematics, Volume 35, Numbers 2-4, 217-241 (2011).

[8] C. Conti, K. Hormann, Polynomial reproduction for univariate subdivision schemes of any arity, Journal of Approximation Theory, Volume 163(4), (2011), 413-437.

[9] C. Conti, L. Romani, Algebraic conditions on non-stationary subdivision symbols for exponential polynomial reproduction, Journal of Computational and Applied Mathematics 236, (2011), 543556

[10] N. Dyn and D. Levin, Subdivision schemes in geometric modelling, Acta Numer. 11, 73-144 (2002).

[11] N. Dyn, J. Gregory, D. Levin, A Butterfly Subdivision Scheme for Surface Interpolation with Tension Control, ACM Trans. on Graphics 9 , 160-169 (1990).

[12] A. F. Rabarison and J. de Villiers, On Bivariate Interpolatory Mask Symbols, Subdivision and Refinable Functions, Approximation Algorithms for Complex Systems, Springer Proceedings in Mathematics, 2011, Volume 3, Part 2, 275-292 (2011).

[13] I. Gohberg, P. Lancaster, L. Rodman, Matrix polynomials, Academic Press Inc., New York, 1982.

[14] R.Q. Jia, Subdivision schemes in $L_{p}$ spaces, Adv. Comput. Math. 3, 309-341, (1995).

[15] R. Q. Jia, Interpolatory subdivision schemes induced by box splines, Appl. Comput. Harmon. Anal. 8, no. 3, 286-292 (2000).

[16] B. Han and R.Q. Jia, Multivariate refinement equations and convergence of subdivision schemes, SIAM J. Math. Anal. 29 1177-1199, (1998).

[17] W. Lawton, S.L. Lee, Zuowei Shen, Stability and orthonormality of multivariate refinable functions, SIAM Journal on Mathematical Analysis, 28, (1997), 999-1014.

[18] M. Newman, Integral matrices, Pure and Applied Mathematics, Vol. 45, Academic Press, New York, 1972.

[19] S. D. Riemenschneider, Z. Shen, Multidimensional interpolatory subdivision schemes, Siam J. Numer. Anal. 34, 2357-2381 (1997).

[20] Hui Ji, S. D. Riemenschneider, Zuowei Shen, Multivariate compactly supported fundamental refinable functions, duals and biorthogonal wavelets, Studies in Applied Mathematics,102, 173204 (1999). 
[21] T. Sauer, Multivariate refinable functions, differences and ideals: a simple tutorial, J. Comput. Appl. Math. 221, no. 2, 447-459 (2008). 